\title{
Economic analysis of Mycobacterium avium subspecies paratuberculosis vaccines in dairy herds
}

\author{
J. Cho, ${ }^{{ }^{1}}$ L. W. Tauer, ${ }^{*}$ Y. H. Schukken, † M. I. Gómez, ${ }^{*}$ R. L. Smith,† Z. Lu,† and Y. T. Grohn† \\ ${ }^{*}$ Charles H. Dyson School of Applied Economics and Management, and \\ †Department of Population Medicine and Diagnostic Sciences, Cornell University, Ithaca, NY 14853
}

\begin{abstract}
Johne's disease, or paratuberculosis, is a chronic infectious enteric disease of ruminants, caused by infection with Mycobacterium avium ssp. paratuberculosis (MAP). Given the absence of a fail-safe method of prevention or a cure, Johne's disease can inflict significant economic loss on the US dairy industry, with an estimated annual cost of over $\$ 200$ million. Currently available MAP control strategies include management measures to improve hygiene, culling MAP serologic- or fecal-positive adult cows, and vaccination. Although the 2 first control strategies have been reported to be effective in reducing the incidence of MAP infection, the changes in herd management needed to conduct these control strategies require significant effort on the part of the dairy producer. On the other hand, vaccination is relatively simple to apply and requires minor changes in herd management. Despite these advantages, only $5 \%$ of US dairy operations use vaccination to control MAP. This low level of adoption of this technology is due to limited information on its cost-effectiveness and efficacy and some important inherent drawbacks associated with current MAP vaccines. This study investigates the epidemiological effect and economic values of MAP vaccines in various stages of development. We create scenarios for the potential epidemiological effects of MAP vaccines, and then estimate economically justifiable monetary values at which vaccines become economically beneficial to dairy producers such that a net present value (NPV) of a farm's net cash flow can be higher than the NPV of a farm using no control or alternative nonvaccine controls. Any vaccination with either low or high efficacy considered in this study yielded a higher NPV compared with a no MAP control. Moreover, high-efficacy vaccines generated an even higher NPV compared with alternative controls, making vaccination economically attractive. Two high-efficacy vaccines were particularly effective in
\end{abstract}

Received July 29, 2011.

Accepted December 10, 2011.

${ }^{1}$ Corresponding author: jc529@cornell.edu
MAP control and NPV maximization. One was a highefficacy vaccine that reduced susceptibility to MAP infection. The other was a high-efficacy vaccine that had multiple efficacies on the dynamics of MAP infection and disease progress. Only one high-efficacy vaccine, in which the vaccine is targeted at reducing MAP shedding and the number of clinical cases, was not economically beneficial to dairy producers compared with an alternative nonvaccine control, when herds were highly infected with MAP.

Key words: Johne's disease, Mycobacterium avium ssp. paratuberculosis vaccine, economic analysis, vaccination

\section{INTRODUCTION}

Johne's disease (JD), or paratuberculosis, is a chronic enteric disease of ruminants caused by infection with Mycobacterium avium ssp. paratuberculosis (MAP). Johne's disease is one of the most serious infectious diseases in dairy cattle given that it produces considerable economic losses and that no cure or fail-safe prevention exists at present. Infection routes of MAP in dairy cattle include intrauterine infection, postpartum infection via fecal-oral contact, and the uptake of MAP-contaminated colostrum and milk (Sweeney, 1996; USDA, 1997; Benedictus et al., 2008). Infection with MAP normally occurs very early in the life of dairy cattle, but clinical symptoms of JD are most commonly seen in adults and include reduced milk production, decreased fertility, decreased BW, and increased mortality (USDA, 1997; Kudahl and Nielsen, 2009; Smith et al., 2009; Aly et al., 2010). Although not all MAP-infected animals develop clinical JD, the production inefficiencies alone can cause significant economic loss for dairy producers (Ott et al., 1999; Groenendaal et al., 2002; Pillars et al., 2009; Raizman et al., 2009).

In the United States in 1997, the annual cost to the dairy industry was estimated to be more than $\$ 200$ million when herd-level MAP prevalence was approximately $22 \%$ (USDA, 1997). This cost has likely risen as the percentage of US dairy herds infected with MAP has increased to at least 68\% (USDA, 2008). Moreover, a potential link between MAP and Crohn disease (Feller 
et al., 2007; Waddell et al., 2008; Hermon-Taylor, 2009) could further increase the cost of MAP to the dairy industry by prompting stricter regulations on dairy production or altering consumption of dairy products (Groenendaal and Zagmutt, 2008). These animal and human health concerns, together with the economic losses associated with MAP, have increased public interest in minimizing the spread of MAP infection and ultimately in eradicating MAP altogether.

In the absence of effective treatment, calf hygiene management and test-and-cull control strategies are usually recommended and practiced for controlling MAP. Calf hygiene management aims to prevent postpartum infection of calves, the most susceptible animals, by providing MAP-free colostrum and milk and avoiding MAP contamination of the calving area. Test-and-cull aims to remove MAP-shedding animals, the main source of MAP infection and economic loss, by testing adults and slaughtering those found to be test-positive. Both control strategies are economically beneficial for dairy producers compared with no control and are reported to be effective in reducing the incidence of MAP infection (Dorshorst et al., 2006; Collins et al., 2010; Lu et al., 2010; Sorge et al., 2010; Cho et al., 2011). However, these 2 control strategies require significant added effort on the part of the dairy producer to achieve effective MAP control. Moreover, test-and-cull often allows a large portion of MAP-infected animals, particularly infected animals shedding low amounts of MAP, to remain in the herd and act as a source of further MAP infection due to the low sensitivity of currently available MAP tests (Collins et al., 2006). The limitations associated with these 2 strategies often result in limited adoption or success of the control and elimination of MAP in dairy herds.

Besides the above control strategies, MAP vaccinations are currently available and reported to be costeffective and provide partial protection by decreasing fecal shedding of MAP and reducing the clinical symptoms of JD (Van Schaik et al., 1996; Kalis et al., 2001; Muskens et al., 2002; Groenendaal and Galligan, 2003; Rosseels et al., 2006). However, vaccination is the least used strategy for controlling MAP (5\% of dairy producers use vaccination as their MAP control strategy, USDA, 2008), because (1) the reported efficacy of the vaccines is varied and inconclusive (Harris and Barletta, 2001; Köhler et al., 2001; Muskens et al., 2002); (2) only one study has investigated the cost-effectiveness of vaccination based on field trial data rather than assumptions (Van Schaik et al., 1996); and (3) there are some inherent drawbacks associated with current MAP vaccines (Harris and Barletta, 2001, Köhler et al., 2001, Muskens et al., 2002). However, the true reason for failure to adopt vaccination has not been studied.
Killed MAP vaccines, which are most commonly used worldwide, have been found to cause crossreactivity with bovine tuberculosis (TB) diagnostics, which might result in false-positive TB test results for MAP-vaccinated animals. Such false-positive TB tests may have a large effect on TB control; therefore, MAP vaccination is limited to farms that are under close monitoring of regulatory agencies. This, together with limited information on their efficacy on MAP control, results in vaccination being used by only $5 \%$ of dairy operations in the United States as a control method for MAP (USDA, 2008). Alternatives such as live vaccines, although generally more effective, have the potential risk of spreading viable MAP and therefore have not been approved in the United States.

Despite such limitations, vaccination has distinct advantages over calf hygiene management and test-andcull. In particular, its ease of application and minimal need for changes in herd management might make it an attractive MAP-control strategy. In addition, several types of improved MAP vaccines, including subunit-based, DNA-based, and recombinant, are in various stages of research, development, and evaluation worldwide to overcome the limitations associated with currently available vaccines and to enhance efficacy in MAP control (Koets et al., 2006; Rosseels and Huygen, 2008; Keeble and Walker, 2009). Although reports on the cost-effectiveness and efficacy of MAP vaccines are not yet fully informed or conclusive, these new developments, together with the aforementioned advantages, make vaccination of increasing interest to the dairy industry. Moreover, in the United States, potential additional costs caused by cross-reactivity between certain MAP vaccines and the TB test have been mitigated under a new federal order that has removed restrictions on herd movement and TB testing obligations, even in states where TB has been found (USDA, 2010). Consequently, investigating the economics and epidemiological consequences of various MAP vaccines can help the dairy industry make informed choices for MAP control.

The objective of this study was to evaluate the economic value of various MAP vaccines in dairy herds based on their effects on MAP control. Because information on the efficacy and availability of vaccines is limited, we first created scenarios for the potential effects of various vaccines on epidemiological progress of MAP infection, and then identify economically justifiable monetary values for them to be economically beneficial to dairy producers. This value is the maximum vaccination cost per dose that dairy producers would be willing to pay such that they are indifferent between applying vaccination and or an alternative control strategy. To do this, we developed a discrete dynamic model that incorporates both economics and 
different epidemiological effects of vaccines on MAP transmission in a dairy herd. This model is coded using a mathematical programming and optimization software and empirically solved for a farm with alternative initial MAP infection levels to take into account a wide array of dairy farm situations.

\section{MATERIALS AND METHODS}

A discrete dynamic model was constructed to evaluate the economic value of various MAP vaccines based on their epidemiological consequences in MAP control. Because MAP infection takes several years to develop into JD, control measures require years to show tangible effectiveness. Therefore, evaluating the economic value of vaccination is best accomplished by using a dynamic model that incorporates (1) the dynamics of MAP transmission within a herd and (2) the net present value (NPV) of a farm's cash flow over a long-term planning duration. The following section presents a MAP transmission model within a dairy herd that incorporates the epidemiological impact of various MAP vaccines. Scenarios representing various MAP vaccine effects are then discussed. Last, an NPV formula is presented for dairy operations, taking into account the economic benefits and costs associated with vaccination.

\section{MAP Transmission Model}

The animal compartment model was developed from a previous multi-group model that described MAP transmission in a dairy herd ( $\mathrm{Lu}$ et al., 2008, 2010; Mitchell et al., 2008; Cho et al., 2011). This animal compartment model is described in Figure 1, and the definitions of symbols are presented in Table 1. In Figure 1, animals within a herd are grouped into discrete and disjoint compartments according to their (1) infection state, (2) age with 6-mo time steps, and (3) vaccination state. Animal infection states of MAP in a dairy herd are classified as susceptible, resistant, transient, latent, low-shedding, and high-shedding. Animals in susceptible and resistant states are noninfected (free of MAP infection), whereas animals in transient, latent, low-shedding, and high-shedding states are infected with MAP. Animals in the transient state do not show signs of JD and shed MAP transiently at a low level shortly after initial MAP infection (Whitlock et al., 2000). Animals in the latent state also do not show signs of JD, and shed no MAP. Animals in the low-shedding state are in a subclinical stage of JD and shed low levels of MAP $\leq 30 \mathrm{cfu} / \mathrm{g}$ (Whitlock et al., 2000), whereas animals in the high-shedding state are potentially showing clinical signs of JD and shed high levels of MAP >30 cfu/g (Whitlock et al., 2000).

Animal age is closely related to susceptibility to infection and duration of infection states. In a dairy herd, animals are typically susceptible to infection up to the age of 12 mo (Collins and Morgan, 1991; Sweeney, 1996; Whitlock and Buergelt, 1996) and then become resistant afterward. Although some animals born to infected dams may be directly infected with MAP via intrauterine infection (Sweeney et al., 1992b), susceptible animals are most likely infected following contact with MAP from infected animals (Sweeney et al., 1992a; Whitlock et al., 2005a) and in contaminated environments (USDA, 1997). These newly infected animals enter the transient state, which often develops within a few days of infection and continues up to 6 mo (Rankin, 1961). Given the duration of susceptible and transient states, animals in this state are typically younger than 18 mo old. The latent state generally occurs following the transient state and continues for a long time, but animals older than 24 mo in this state may begin to enter the low-shedding state and then the high-shedding state as the disease progresses.

Although some variation exists in the duration of infection states, 14 different infection and age classifications, $i \in \boldsymbol{I}=\{1, \ldots, 14\}$, were defined to describe the above relationship between MAP infection progress and animal age: calves 0 to 6 mo old in susceptible ( $i$ $=1$ ) or transient state $(i=2)$; calves 6 to 12 mo old in susceptible $(i=3)$, transient $(i=4)$, or latent $(i=$ $5)$ state; heifers 12 to 18 mo old in resistant $(i=6)$, transient $(i=7)$, or latent $(i=8)$ state; heifers 18 to 24 mo old in resistant $(i=9)$ or latent $(i=10)$ state; and adult cows older than 24 mo in resistant $(i=11)$, latent $(i=12)$, low-shedding $(i=13)$, or high-shedding $(i=14)$ state.

To examine the effect of MAP vaccines, animals were further classified into their vaccination state, unvaccinated or vaccinated, the number of which are represented respectively as $X_{i}$ and $V_{i}$ in Figure 1 for 14 different infection and age classifications $i \in \boldsymbol{I}=\{1, \ldots, 14\}$. In this model, some newborn calves entered the herd as noninfected, susceptible calves $\left(X_{1}\right.$ and $\left.V_{1}\right)$, whereas others $\left(X_{2}\right.$ and $\left.V_{2}\right)$ directly entered the transient state at the time of birth via intrauterine infection, which is represented by rate $\gamma$, as shown in Figure 1. All calves, heifers, and adult cows were assumed to exit the herd at rates $\mu_{\mathrm{c}}, \mu_{\mathrm{h}}$, and $\mu_{\mathrm{a}}$ in Figure 1, respectively. In addition, all high-shedding cows exited the herd at an additional rate $\alpha$ due to clinical symptoms of JD. All remaining animals in each compartment for the current period were assumed to move to an adjacent compartment for the next period due to aging $\left(\chi, \varepsilon, \rho_{\mathrm{c}}, \rho_{\mathrm{h}}\right)$, 


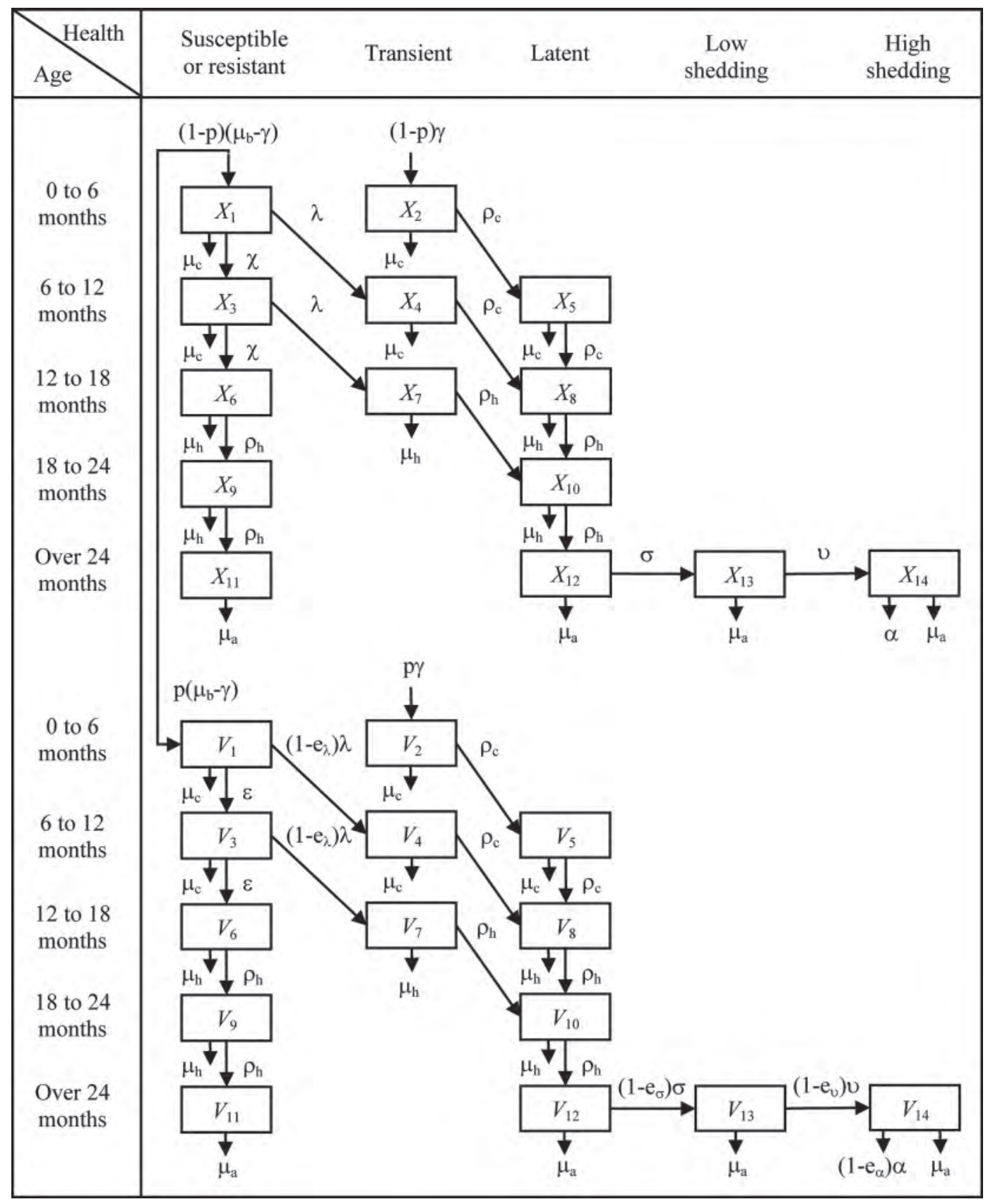

Figure 1. Animal compartment model, with the top segment representing no vaccination and the bottom segment representing vaccination across animal groups. The variables $X_{i}$ and $V_{i}$ represent the number of unvaccinated $\left(X_{i}\right)$ and vaccinated $\left(V_{i}\right)$ animals in different infection (top row) and age (left column) classifications.

infection with $\operatorname{MAP}(\lambda)$, or infection progress $\left(\rho_{\mathrm{c}}, \rho_{\mathrm{h}}\right.$, $\sigma, v)$. In the model, the rates $\rho_{\mathrm{c}}$ and $\rho_{\mathrm{h}}$ represent an animal movement for infected calves and heifers, respectively, due to aging or the infection progress. For example, all calves 6 to $12 \mathrm{mo}$ old in the latent state became heifers 12 to $18 \mathrm{mo}$ old in the latent state for the next period due to aging. Thus, the rate $\rho_{\mathrm{c}}$ representing this movement is $1-\mu_{\mathrm{c}}$, where $\mu_{\mathrm{c}}$ is the death rate of calves. On the other hand, all calves 6 to 12 mo old in the transient state became heifers 12 to 18 mo old in the latent state for the next period due to infection progress. Therefore, this movement can also be represented by $\rho_{\mathrm{c}}=1-\mu_{\mathrm{c}}$. An arrow in Figure 1 indicates this movement. The equations of motion that represent the animal compartment model of Figure 1 are shown in the Appendix. 
Table 1. Definition of symbols in the animal compartment model describing animal movements and Mycobacterium avium ssp. paratuberculosis (MAP) transmission within a herd

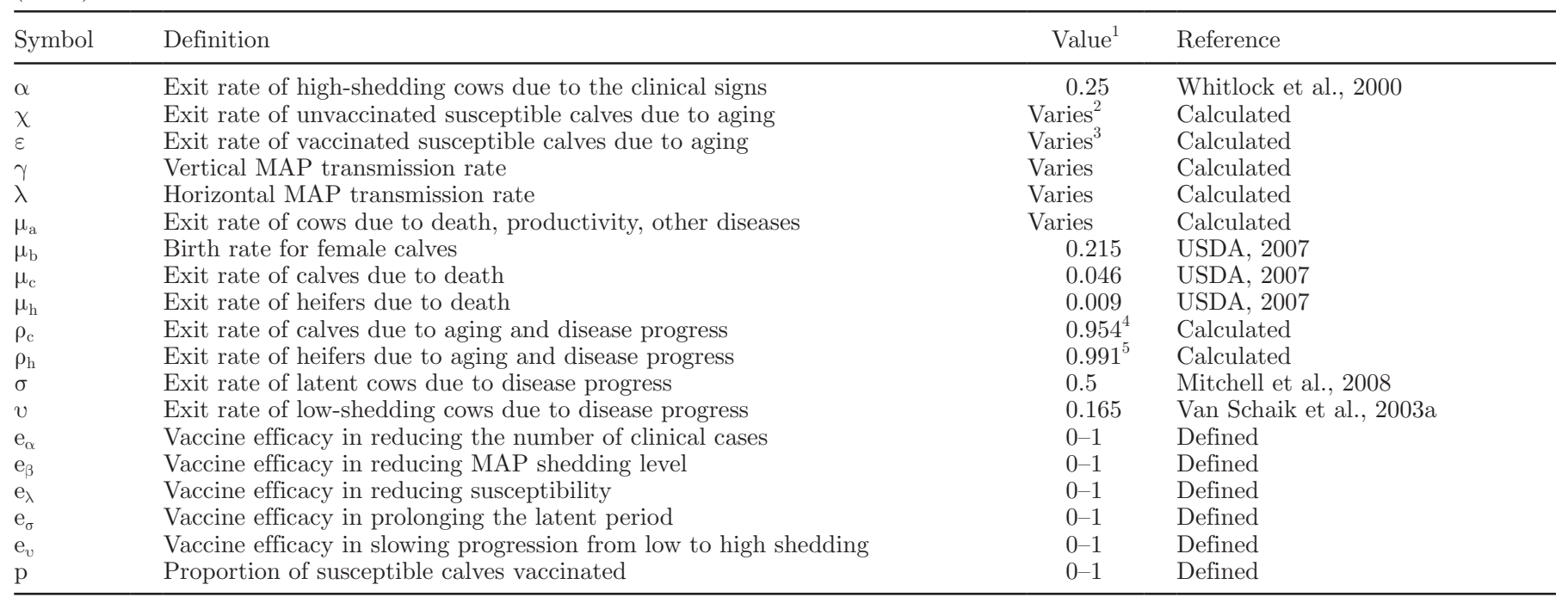

${ }^{1}$ Values are on a 6 -mo basis.

${ }^{2} \chi=1-\mu_{\mathrm{c}}-\lambda$ represents animal movements for unvaccinated susceptible calves due to aging.

${ }^{3} \varepsilon=1-\mu_{\mathrm{c}}-\left(1-\mathrm{e}_{\lambda}\right) \lambda$ represents animal movements for unvaccinated susceptible calves due to aging.

${ }^{4} \rho_{\mathrm{c}}=1-\mu_{\mathrm{c}}=0.954$ represents animal movements for infected calves due to either aging or infection progress: all calves 6 to 12 mo old in the latent state become heifers 12 to 18 mo old in the latent state for the next time period due to aging. Thus, the rate $\rho_{\mathrm{c}}$ representing this movement is $1-\mu_{\mathrm{c}}$ where $\mu_{\mathrm{c}}$ is the death rate of calves. On the other hand, all calves 6 to 12 mo old in the transient state become heifers 12 to 18 mo old in the latent state for the next period due to infection progress. Therefore, this movement can also be represented by $\rho_{\mathrm{c}}=1-\mu_{\mathrm{c}}$.

${ }^{5} \rho_{\mathrm{h}}=1-\mu_{\mathrm{h}}=0.991$ represents animal movements for infected heifers due to either aging or infection progress, with the same reason as $\rho_{\mathrm{c}}$ representing animal movements for infected calves due to either aging or infection progress.

Because revaccination or boosting has been reported to be ineffective in enhancing an animal's ability to resist MAP infection (Harris and Barletta, 2001), only single vaccination of newborn calves is modeled in this study. In Figure 1, a proportion of newborn calves was vaccinated with parameter $\mathrm{p}(0 \leq P \leq 1)$, where $P=0$ indicates that no calves are vaccinated, whereas $P=1$ indicates that all calves are vaccinated. Once newborn calves are vaccinated, they move along the vaccinated animal compartments at the bottom of Figure 1, whereas unvaccinated newborn calves move along the unvaccinated animal compartments at the top of Figure 1. For the sake of model brevity, all newborn calves are assumed to be vaccinated $(P=1)$ when producers implement vaccination as a control strategy for MAP.

The MAP infection routes of both intrauterine infection (vertical transmission) and postpartum infection (horizontal transmission) are considered in Figure 1. Vertical MAP transmission due to infected dams determines the number of transient calves 0 to 6 mo old $\left(X_{2}\right.$ and $V_{2}$ ) and is formulated in the vertical transmission rate $\gamma$ in Figure 1:

$$
\gamma(t)=\mu_{b}\left\{\begin{array}{l}
\gamma_{12}\left[X_{12}(t)+V_{12}(t)\right]+\gamma_{13}\left[X_{13}(t)+V_{13}(t)\right] \\
+\gamma_{14}\left[X_{14}(t)+V_{14}(t)\right]
\end{array}\right\}
$$

where $\mu_{b}$ is the birth rate of female calves, and $\gamma_{12}, \gamma_{13}$, and $\gamma_{14}$ (Table 2) are the proportions of calves infected in utero by latent $\left(X_{12}, V_{12}\right)$, low-shedding $\left(X_{13}, V_{13}\right)$, or high-shedding $\left(X_{14}, V_{14}\right)$ dams at time $t$, respectively. These parameters $\left(\gamma_{12}, \gamma_{13}\right.$, and $\left.\gamma_{14}\right)$ may vary for unvaccinated and vaccinated infected dams. However, given the lack of information on these potential differences, the intrauterine transmission parameters were assumed identical for both unvaccinated and vaccinated infected dams.

Susceptible calves $\left(X_{1}, X_{3}, V_{1}, V_{3}\right)$ may be infected by horizontal MAP transmission via fecal-oral contact and the uptake of MAP-contaminated colostrum and milk: infection by transiently shedding calves $\left(X_{2}\right.$, $X_{4}, X_{7}, V_{2}, V_{4}, V_{7}$ ) at the same housing site (calf-calf transmission) or by adult shedding cows $\left(X_{13}, X_{14}, V_{13}\right.$, $\left.V_{14}\right)$ through direct fecal-oral transmission and indirect transmission via contaminated colostrum and milk (cow-calf transmission). To keep the modeling process manageable, 3 transmission rates (Table 2) were used to represent the multiple transmission routes: calfcalf transmission $\left(\beta_{T r}\right)$ and cow-calf transmission by low- $\left(\beta_{13}\right)$ and high- $\left(\beta_{14}\right)$ shedding cows. The force of infection for unvaccinated susceptible calves $\left(X_{1}, X_{3}\right)$ is formulated as $\lambda$ in Figure 1: 
Table 2. Definition of parameters in vertical and horizontal infection transmission rate formulas

\begin{tabular}{llll}
\hline Symbol & Definition & Value ${ }^{1}$ & Reference \\
\hline$\beta_{T r}$ & Transmission rate between transient animals and susceptible calves & 0.5 & Lu et al., 2008 \\
$\beta_{13}$ & Transmission rate between low-shedders and susceptible calves & Lu et al., 2008 \\
$\beta_{14}$ & Transmission rate between high-shedders and susceptible calves & 5.0 & Lu et al., 2008 \\
$\gamma_{12}$ & Portion of newborn calves infected at birth by latently infected dams & 0.15 & Sweeney et al., 1992b \\
$\gamma_{13}$ & Portion of newborn calves infected at birth by low-shedding dams & 0.15 & Whitlock et al., 2005a \\
$\gamma_{14}$ & Portion of newborn calves infected at birth by high-shedding dams & 0.17 & Whitlock et al., 2005b \\
\hline
\end{tabular}

${ }^{1}$ Values are on a 6-mo basis.

$\lambda(t)=\left(\begin{array}{l}\beta_{T r}\left[X_{2}(t)+X_{4}(t)+X_{7}(t)\right]+\beta_{13} X_{13}(t) \\ \left.+\beta_{14} X_{14}(t)+\left(1-e_{\beta}\right)\left\{\begin{array}{l}\beta_{T r}\left[V_{2}(t)+V_{4}(t)+V_{7}(t)\right] \\ +\beta_{13} V_{13}(t)+\beta_{14} V_{14}(t)\end{array}\right\}\right)\end{array}\right) / N(t)$,

where $N(t)$ is the total number of animals in a farm, and potential vaccine efficacy in reducing fecal shedding level is represented by parameter $e_{\beta}\left(0 \leq e_{\beta} \leq\right.$ $1)$, such that $e_{\beta}=0$ indicates that no vaccination is implemented or a vaccine has no decreasing effect on MAP shedding, whereas $e_{\beta}=1$ indicates that a vaccine completely prevents MAP shedding. On the other hand, the force of infection for vaccinated susceptible calves $\left(V_{1}, V_{3}\right)$ is modeled as $\left(1-e_{\lambda}\right) \lambda$ in Figure 1 to represent potential vaccine efficacy in reducing susceptibility $e_{\lambda}\left(0 \leq e_{\lambda} \leq 1\right)$, such that $e_{\lambda}=0$ indicates that a vaccine has no reducing effect on susceptibility to infection, whereas $e_{\lambda}=1$ indicates that a vaccine completely prevents postpartum MAP infection.

In addition to reducing MAP shedding levels and susceptibility to infection, 3 other vaccine efficacies were considered for current or next-generation vaccines. These efficacies included prolonging the latent period of infected animals by delaying fecal shedding, slowing the progression of infectious animals from low to high shedding, or decreasing the number of clinical JD cases (Harris and Barletta, 2001; Rosseels et al., 2006; Rosseels and Huygen, 2008; Romano and Huygen, 2009). These potential efficacies are represented by the parameters $e_{\sigma}, e_{v}$, and $e_{\alpha}$ in Figure 1, respectively.

\section{Scenarios of MAP Vaccine Efficacy}

Five possible efficacies are assumed for MAP vaccines (Figure 1): reduction of susceptibility $\left(e_{\lambda}\right)$; reduction of MAP shedding level $\left(e_{\beta}\right)$; slower progression from latency to low shedding $\left(e_{\sigma}\right)$; slower progression from low to high shedding $\left(e_{v}\right)$; and a slower progression to clinical JD status $\left(e_{\alpha}\right)$. These efficacies range from 0 (not effective at all) to 1 (fully effective). Because information on MAP vaccine efficacies is limited, specific values for the parameters listed above are not fixed; instead, each efficacy is assigned a representative value of 0.3 (relatively low efficacy) or 0.9 (relatively high efficacy). The economic values of vaccines with these 2 representative values can be used as reference values for those vaccines having different levels of efficacy.

The 8 scenarios in Table 3 , which comprise various combinations of vaccine efficacies, were developed for representing the potential effect of different types of vaccines on the epidemiological process of MAP. These scenarios allow us to compare different epidemiological effects of current and possibly next-generation vaccines on MAP control. Scenarios L-S and H-S represent vaccines with low and high efficacy levels, respectively, which specifically target reduction of susceptibility (perhaps the most desirable feature of MAP vaccines). Scenarios L-LC and H-LC represent vaccines with low and high efficacy values, respectively, for the vaccine effects reported in previous studies (Van Schaik et al., 1996; Kalis et al., 2001; Muskens et al., 2002; Groenendaal and Galligan, 2003; Rosseels et al., 2006), including decrease of fecal MAP shedding and reduction of clinical JD symptoms. Scenarios L-OP, H-OP, L-LOPC, and H-LOPC represent vaccines having other multiple efficacies that could be produced by some of the currently available or next-generation vaccines.

\section{Net Present Value}

Given a set of epidemiological constraints, the producer objective is to maximize the expected NPV from the sales of milk and cull cows for slaughter by deciding upon a choice of vaccination in Table 3. Epidemiological constraints are the equations of motion in Appendix 1 that describe the epidemiological process of Johne's disease described in Figure 1. Although not all infected animals show clinical JD symptoms, this study assumed that animals in the low-shedding state showed decreased milk production and BW. As these animals progressed to the high-shedding state, they were assumed to show considerable reduction in milk production and BW and have a higher mortality rate. The expected NPV of a producer's net cash flow from the sales of milk and cull cows for slaughter is specified as follows: 
Table 3. Scenarios of Mycobacterium avium ssp. paratuberculosis (MAP) vaccine efficacy

\begin{tabular}{|c|c|c|}
\hline Scenario & Description & Value $^{1}$ \\
\hline $\begin{array}{l}\mathrm{L}-\mathrm{OP} \\
\mathrm{H}-\mathrm{OP}\end{array}$ & Vaccine delays the onset of low shedding and slows the progression from low to high shedding & $\begin{array}{l}\mathrm{e}_{\sigma}=\mathrm{e}_{v}=0.3 \\
\mathrm{e}_{\sigma}=\mathrm{e}_{v}=0.9\end{array}$ \\
\hline $\begin{array}{l}\text { L-LOPC } \\
\text { H-LOPC }\end{array}$ & $\begin{array}{l}\text { Vaccine reduces MAP shedding level, delays the onset of low shedding and progression } \\
\text { from low to high shedding, and decreases the number of clinical JD cases }\end{array}$ & $\begin{array}{l}\mathrm{e}_{\beta}=\mathrm{e}_{\sigma}=\mathrm{e}_{v}=\mathrm{e}_{\alpha}=0.3 \\
\mathrm{e}_{\beta}=\mathrm{e}_{\sigma}=\mathrm{e}_{v}=\mathrm{e}_{\alpha}=0.9\end{array}$ \\
\hline
\end{tabular}

${ }^{1} e_{\alpha}$ represents vaccine efficacy in reducing the number of clinical cases; $e_{\beta}$ represents vaccine efficacy in reducing MAP shedding level; $e_{\lambda}$ represents vaccine efficacy in reducing susceptibility; $\mathrm{e}_{\sigma}$ represents vaccine efficacy in prolonging the latent period; $\mathrm{e}_{v}$ represents vaccine efficacy in slowing progression from low to high shedding.

$$
\begin{gathered}
N P V=\sum_{t=1}^{T-1} \frac{1}{(1+r)^{t}}\left\{\begin{array}{l}
{\left[P_{\text {milk }} Q_{\text {milk }}+P_{\text {cull }} Q_{\text {cull }} \mu_{a}(t)\right]} \\
{\left[X_{11}(t)+X_{12}(t)+V_{11}(t)+V_{12}(t)\right]} \\
+P_{\text {milk }} Q_{\text {milk }}\left(1-\varphi_{L}\right)\left[X_{13}(t)+V_{13}(t)\right] \\
+P_{\text {milk }} Q_{\text {milk }}\left(1-\varphi_{H}\right)\left[X_{14}(t)+V_{14}(t)\right] \\
+P_{\text {cull }} Q_{\text {cull }}\left(1-\zeta_{L}\right)\left[X_{13}(t)+V_{13}(t)\right] \\
\mu_{a}(t)+P_{\text {cull }} Q_{\text {cull }}\left(1-\zeta_{H}\right) \\
{\left[X_{14}(t)+V_{14}(t)\right] \mu_{a}(t)} \\
-C_{\text {calf }} N_{\text {calf }}(t)-C_{\text {heifer }} N_{\text {heifer }}(t) \\
-C_{\text {cow }} N_{\text {cow }}(t)-M_{\text {vaccine }}(t) \mu_{b} N_{\text {cow }}(t)
\end{array}\right\} \\
+\frac{1}{(1+r)^{T}}\left\{\begin{array}{l}
P_{\text {cull }} Q_{\text {cull }} N_{\text {cow }}(T) \\
+P_{\text {sale }}\left[N_{\text {calf }}(T)+N_{\text {heifer }}(T)\right]
\end{array}\right\}
\end{gathered}
$$

In equation [3], resistant $\left(X_{11}\right.$ and $\left.V_{11}\right)$ and latent $\left(X_{12}\right.$ and $\left.V_{12}\right)$ cows were expected to have normal milk production $\left(Q_{\text {milk }}\right)$ and BW $\left(Q_{\text {cull }}\right)$, low-shedding cows $\left(X_{13}\right.$ and $V_{13}$ ) and high-shedding cows (X14 and V14), due to the disease, were expected to have lower milk production, $\left(1-\varphi_{L}\right) Q_{\text {milk }}$ and $(1-\varphi H) Q_{\text {milk }}$, respectively, and lower BW, $\left(1-\zeta_{L}\right) Q_{\text {cull }}$ and $\left(1-\zeta_{H}\right) Q_{\text {cull }}$, respectively, where the parameters $\varphi_{L}, \varphi_{H}, \zeta_{L}$, and $\zeta_{H}$ represent milk production and BW reductions due to JD (Table 4). In addition to milk and BW losses, high-shedding cows could exit the herd due to clinical JD symptoms at a net value of zero.

The entire herd was assumed to be liquidated at the beginning of the terminal year $(T=50)$. For the sake of model brevity, all remaining cows in the terminal years were sold at the price of healthy cows. This is a reasonable assumption given that, with effective controls, no cows will show clinical symptoms of JD at the end of the 50-yr period. Young stock were all sold at the price of 1-yr-old animals, the average age of young stock. The variables and parameters in equation [3] are presented in Figure 1 (animal compartments) and Table $1\left(\mu_{a}, \mu_{b}\right.$, $\mu_{c}$, and $\mu_{h}$ ) and Table 4 (all other parameters).

Because information on retail price of the vaccines is unavailable, equation [3] includes a variable $\left(M_{\text {vaccine }}\right)$ representing the maximum vaccination cost per dose (MVC) for newborn calves. This MVC is determined endogenously in the optimization process by equating the NPV under vaccination to the NPV under an alternative control strategy: (1) the no-control alternative or (2) the best nonvaccine alternative. This allows us to obtain the uppermost cost level of vaccination, or MVC, that the farmer would be willing to pay for vaccination in order for them to be indifferent between applying vaccination and either alternative control strategy, as illustrated in Figure 2. In other words, a vaccination that cost less than the MVC generates a higher NPV to the farm than the NPV obtained using the no-control alternative or the best nonvaccine alternative. A positive MVC implies a positive gross return that can be shared by a dairy producer (profit and implementation cost, if any) and a vaccine company (profit and production cost). Conversely, a negative MVC implies that the vaccination is economically unattractive to a dairy producer because it generates a lower NPV than the no-control or the best nonvaccine alternative cases.

The NPV with the best nonvaccine alternative were obtained from our earlier study (Cho et al., 2011). This previous study used the unvaccinated animal compartments at the top of Figure 1 to examine the costeffectiveness of various MAP control options over the 50 -yr simulation period, which included (1) improved hygiene, (2) advanced hygiene, (3) test-and-cull using annual fecal culture test, (4) test-and-cull using annual ELISA test, (5) test-and-cull using biannual fecal culture test, (6) test-and-cull using biannual ELISA test, (7) improved hygiene with test-and-cull using annual 
Table 4. Definition of variables and parameters used in the net present value equation

\begin{tabular}{llll}
\hline Rate & Description & Value $^{1}$ & Reference \\
\hline $\mathrm{C}_{\text {calf }}$ & Base operating cost of raising a calf & $\$ 395.00^{2}$ & Karszes et al., 2008 \\
$\mathrm{C}_{\text {cow }}$ & Base operating cost of raising a cow & $\$ 1231.46$ & USDA, 2003-2007 \\
$\mathrm{C}_{\text {heifer }}$ & Base operating cost of raising a heifer & $\$ 395.00^{2}$ & Karszes et al., 2008 \\
$\mathrm{M}_{\text {vaccine }}$ & Maximum vaccination cost per dose & Varies & Calculated \\
$\mathrm{N}_{\text {calf }}$ & Number of calves & Varies & Calculated \\
$\mathrm{N}_{\text {cow }}$ & Number of cows & 100 & Calculated \\
$\mathrm{N}_{\text {heifer }}$ & Number of heifers & Varies & Calculated \\
$\mathrm{P}_{\text {cull }}$ & Cull-cow price per kg & $\$ 1.0556$ & USDA, 2003-2007 \\
$\mathrm{P}_{\text {milk }}$ & Milk price per kg & $\$ 0.3393$ & USDA, 2003-2007 \\
$\mathrm{P}_{\text {sale }}$ & Sale price of a one year old animal & $\$ 867^{3}$ & Karszes et al., 2008 \\
$\mathrm{Q}_{\text {cull }}$ & Average cull cow weight & $680.4 \mathrm{~kg}$ & USDA, 2003-2007 \\
$\mathrm{Q}_{\text {milk }}$ & Average milk production per cow & $4408.7 \mathrm{~kg}$ & USDA, 2003-2007 \\
$\mathrm{r}$ & Discount rate & 0.02 & Assumed \\
$\mathrm{T}$ & Total follow up time of a dairy farm & 100 & Assumed \\
$\varphi_{\mathrm{H}}$ & Production adjustment factor for high-shedders & $0.1^{4}$ & Groenendaal et al., 2002 \\
$\varphi_{\mathrm{L}}$ & Production adjustment factor for low-shedders & $0.05^{4}$ & Groenendaal et al., 2002 \\
$\zeta_{\mathrm{H}}$ & Cull-weight adjustment factor for high-shedders & 0.1 & Assumed \\
$\zeta_{\mathrm{L}}$ & Cull-weight adjustment factor for low-shedders & 0.05 & Assumed \\
\hline
\end{tabular}

${ }^{1}$ Values are on a 6 -mo basis.

${ }^{2}$ For the sake of simplicity, all heifer-raising operating costs for $2 \mathrm{yr}, \$ 1,580$ per heifer, are evenly assigned to the calf- and heifer-rearing activities.

${ }^{3}$ Sale price of a 1-yr-old animal is assumed identical to total cost of raising replacement heifer up to $1 \mathrm{yr}$.

${ }^{4}$ Production reduction due to Johne's disease has been reported to be 5 to $20 \%$.

fecal culture test, (8) improved hygiene with test-andcull using annual ELISA test, (9) improved hygiene with test-and-cull using biannual fecal culture test, (10) improved hygiene with test-and-cull using biannual
ELISA test, (11) advanced hygiene with test-and-cull using annual fecal culture test, (12) advanced hygiene with test-and-cull using annual ELISA test, (13) advanced hygiene with test-and-cull using biannual fecal

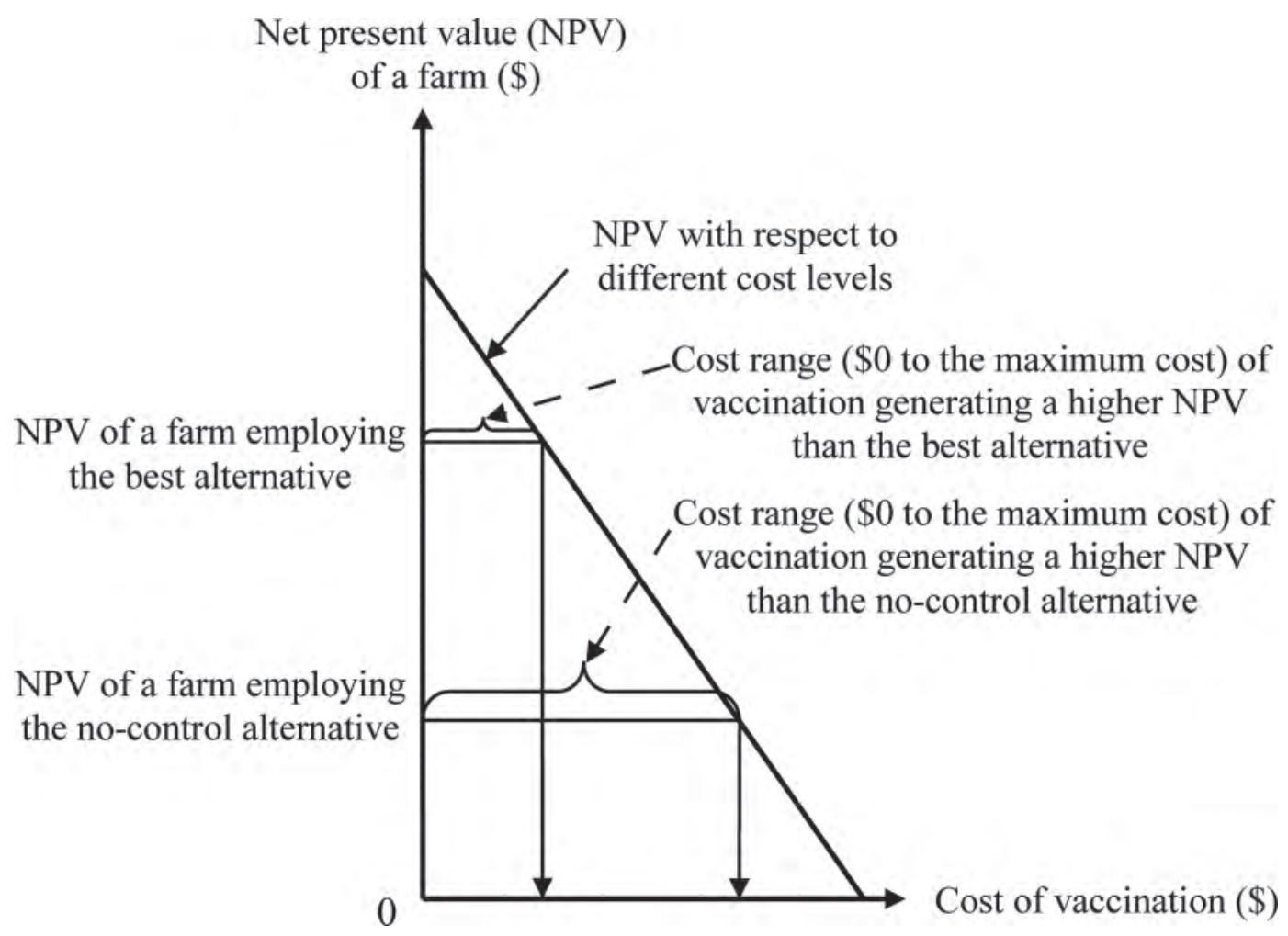

Figure 2. Maximum cost level of a vaccination at which farmers are indifferent between applying vaccination and either the no-control alternative or the best control alternative (improved hygiene with test-and-cull using annual fecal culture test). NPV $=$ net present value. 
culture test, and (14) advanced hygiene with test-andcull using biannual ELISA test.

An earlier study (Cho et al., 2011) utilized the same parameter values for the animal compartment model (Figure 1) and NPV equations (equation 3) described in this study, with the exception that the present study assumed a fixed herd size of 100 cows, whereas the previous study allowed herd size variation of 80 cows (minimum herd requirement) to 100 cows (maximum farm capacity), which allowed heavy culling of animals for the test-and-cull strategy. The results of the previous study showed that the most cost-effective control option for MAP was improved hygiene management and test-and-cull using an annual fecal culture test. Therefore, we used this control option as the best alternative to vaccination, which is the focus of this study.

Because a mean true prevalence level of $10 \%$ MAP infection within a dairy herd is commonly assumed (Wells et al., 2002; Van Schaik et al., 2003b; Dorshorst et al., 2006), 2 initial MAP infection levels (10\% and $20 \%$ ) were considered for the baseline farm to account for the majority of dairy farm situations. Each infection level represents a percentage of MAP-infected adult cows (sum of latent, low-shedding, and high-shedding cows) per all adult cows in a herd. These initial MAP infection levels were obtained from a simulation of unvaccinated animal compartments at the top of Figure 1: an initial infection distribution among unvaccinated animal groups was simulated for a farm with an initial herd of 99 noninfected cows and 1 latently infected cow, with no control implemented. The initial conditions for the state variables for a farm with 2 different MAP infection levels were drawn from time points in this simulation that matched the desired infection level. These initial conditions are identical to those used in Cho et al. (2011).

The model described in this section is coded using the General Algebraic Modeling System (version 22.5, GAMS Development Corp., Washington, DC) and empirically solved for a farm with 2 possible MAP infection levels for a long-term, 50-yr simulation period. The result of this optimization would allow identification of both epidemiological consequences of the 8 vaccination options and the MVC value that makes a certain vaccination option economically more attractive in the long term, compared with no control or the best alternative.

\section{RESULTS AND DISCUSSION}

In the absence of MAP control, simulation of unvaccinated animal compartments (the top of Figure 1) showed that MAP prevalence increased continuously (Figure 3), as reported in previous studies (Groenendaal et al., 2002; Groenendaal and Galligan, 2003). In Fig-

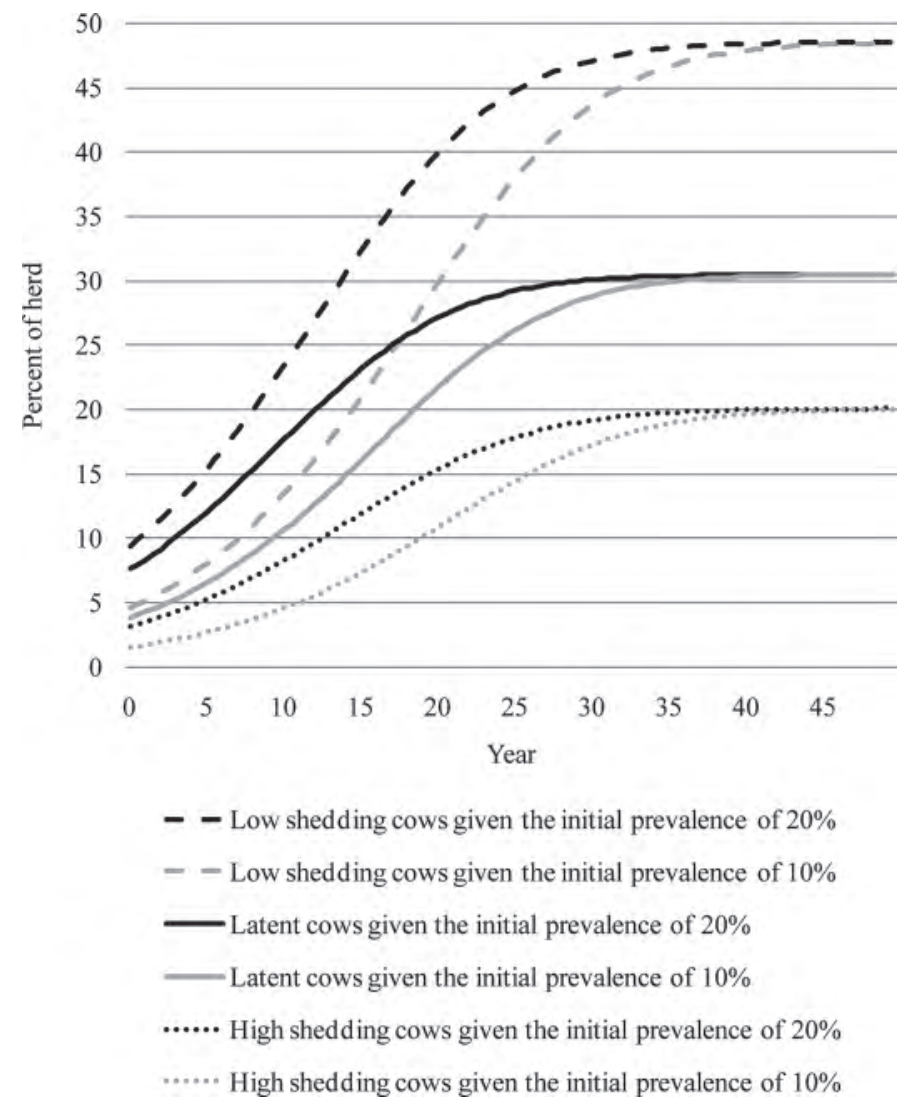

Figure 3. Dynamics of Mycobacterium avium ssp. paratuberculosis (MAP) infection without control: the portions of latent, low-shedding, and high-shedding adult cows in the herd with 100 cows and initial MAP prevalence of 10 and $20 \%$ (vertical axis).

ure 3, the portion of cows in each MAP infection state varies because the duration of each MAP infection state is different. In addition, the marginal increase in the number of cows in each infection state in Figure 3 decreases with time because the number of susceptible animals decreases as the number of infected animals increases with time, given no control.

With any type of low-efficacy vaccines, elimination of MAP was not observed in the 50-yr simulation period given initial infection levels of 10 or $20 \%$ (Table 5; MAP is considered to be eliminated when its prevalence rate is $<1 \%$ ). Only a low-efficacy vaccine with multiple effects on the dynamics of MAP infection and progress (scenario L-LOPC) would control MAP at a constant rate after $5 \mathrm{yr}$ of vaccination (Figure 4). This low-efficacy vaccine was targeted at reducing MAP shedding level, delaying the onset of shedding and progression from low to high shedding, and decreasing the number of clinical JD cases. All other low-efficacy vaccines resulted in an increase in MAP prevalence (Figure 4), especially those targeted at reducing the MAP shedding level and the number of clinical JD cases (scenario L-LC), and 
Table 5. Expected elimination period of Mycobacterium avium ssp. paratuberculosis (MAP) for a herd with vaccination and the initial infection level of $10 \%$ and $20 \%$

\begin{tabular}{lcc}
\hline & \multicolumn{2}{c}{ Expected elimination $^{2}$ period of MAP } \\
\cline { 2 - 3 } Scenario $^{1}$ & $\begin{array}{c}\text { Infection level } \\
\text { of } 10 \%\end{array}$ & $\begin{array}{c}\text { Infection level } \\
\text { of } 20 \%\end{array}$ \\
\hline L-S & Never & Never \\
H-S & 10.5 yr & 13 yr \\
L-LC & Never & Never \\
H-LC & 22.5 yr & 26 yr \\
L-OP & Never & Never \\
H-OP & 29 yr & 34 yr \\
L-LOPC & Never & Never \\
H-LOPC & 17.5 yr & 20 yr \\
\hline
\end{tabular}

${ }^{1}$ The notation L-S and H-S represent a low- (L-S) and high-efficacy (H-S) vaccine, in which the vaccine is targeted at decreasing susceptibility of susceptible calves. L-LC and H-LC represent a low- (L-LC) and high-efficacy (H-LC) vaccine, in which the vaccine is targeted at reducing shedding level and the number of clinical Johne's disease cases. L-OP and H-OP represent a low- (L-OP) and high-efficacy $(\mathrm{H}-$ $\mathrm{OP})$ vaccine, in which the vaccine is targeted at delaying the onset of low shedding and slowing the progression from low to high shedding. L-LOPC and H-LOPC represent a low- (L-LOPC) and high-efficacy (H-LOPC) vaccine, in which the vaccine is targeted at reducing MAP shedding level, delaying the onset of low shedding and progression from low to high shedding, and decreasing the number of clinical Johne's disease cases. ${ }^{2} \mathrm{MAP}$ is considered eliminated when its prevalence rate is less than $1 \%$, which implies that total number of MAP-infected cows is less than 1 given a fixed herd size of 100 cows.

delaying the onset of MAP shedding and slowing the progression from low to high shedding states (scenario $\mathrm{L}-\mathrm{OP}$ ). These vaccination scenarios resulted in a rapid increase of MAP prevalence because they had only partial and limited effects on reducing sources of MAP infection, which results in a elimination process slower than the MAP infection process within a herd.

In sharp contrast, all high-efficacy vaccines successfully eliminated MAP within 29 and $34 \mathrm{yr}$ for a herd with initial infection levels of 10 and $20 \%$, respectively (Table 5). However, none of these high-efficacy vaccines eliminated MAP faster than the best alternative of improved hygiene management and test-and-cull using an annual fecal culture test, which eliminated MAP from the herd within 8 yr for both MAP prevalence levels (Cho et al., 2011). The effect of these high-efficacy vaccines on the dynamics of MAP infection (Figure 5 ) suggests that the most effective vaccine scenario for eliminating MAP was a high-efficacy vaccine that reduced susceptibility of susceptible calves (scenario H-S), which eliminated MAP within 10.5 yr (initial prevalence of 10\%) and $13 \mathrm{yr}$ (initial prevalence of $20 \%$ ), as shown in Table 5 and Figure 5. This finding implies that the prevention effect is the most useful possible effect of vaccines in MAP control.

The second most effective vaccine scenario for eliminating MAP was a high efficacy vaccine with multiple effects on the dynamics of MAP infection and progress (scenario H-LOPC), eliminating MAP within 17.5 yr (initial prevalence of $10 \%$ ) or $20 \mathrm{yr}$ (initial prevalence of 20\%), as shown in Table 5 and Figure 5. This high-efficacy vaccine was targeted at reducing MAP shedding level, delaying the onset of shedding and progression from low to high shedding, and decreasing the number of clinical JD cases. Given the multiple efficacies associated with this vaccine, a large portion of infected cows were nonclinically infected (latent cows), which does not cause economic loss. In addition, the aforementioned low-efficacy vaccine (scenario L-LOPC in Figure 4) with the same epidemiological effect as this high-efficacy vaccine in scenario H-LOPC, was the only low-efficacy vaccine that controlled MAP at a constant rate. Therefore, dairy producers might want to control MAP using this low-efficacy vaccine (scenario L-LOPC, Figure 4) among all available vaccines when only lowefficacy vaccines are available, whereas they might want to control MAP using a high-efficacy vaccine (scenario $\mathrm{H}-\mathrm{S}$ in Figure 4) that reduces the susceptibility of eligible calves if high-efficacy vaccines become available.

Two other high-efficacy vaccines also eliminated MAP. One vaccine was targeted at reducing both the shedding level and the number of clinical JD cases (scenario H-LC) within 22.5 yr (initial prevalence of $10 \%$ ) and $26 \mathrm{yr}$ (initial prevalence of $20 \%$ ), as shown in Table 5 and Figure 5. The other was targeted at delaying the onset of shedding and slowing the progression from low to high shedding (scenario $\mathrm{H}-\mathrm{OP}$ ) within $29 \mathrm{yr}$ (initial prevalence of $10 \%$ ) and $34 \mathrm{yr}$ (initial prevalence of 20\%), also shown in Table 5 and Figure 5. Although the vaccine in scenario $\mathrm{H}-\mathrm{OP}$ took longer to eliminate MAP than that in scenario $\mathrm{H}-\mathrm{LC}$, the portion of latent cows among all infected cows in scenario $\mathrm{H}-\mathrm{OP}$ was much larger. The fact that latent cows do not cause economic loss, coupled with the epidemiological consequences of the second most effective vaccine in scenario H-LOPC, implies that prolongation of latency and slowed progression from low to high shedding may be the more economically beneficial effect of vaccination rather than reductions of MAP shedding level and the number of clinical JD cases. However, given the short MAP elimination period associated with scenario $\mathrm{H}-\mathrm{OP}$, the relative economic benefit of the vaccines in scenarios H-LC and H-OP is not as clear when considering epidemiological consequences alone. The epidemiological effects of low- and high-efficacy vaccinations in Figures 4 and 5 suggest that the level of efficacy of vaccines is a more important feature of vaccination than either MAP infection level within a herd or the effects of a vaccine on the dynamics of MAP infection. In conclusion, any high-efficacy vaccine showed considerably better effects on MAP control compared with any low-efficacy vaccine. 
- Latent ---- Low ……….. High


\section{L-LOPC with $10 \%$ Initial Infection}

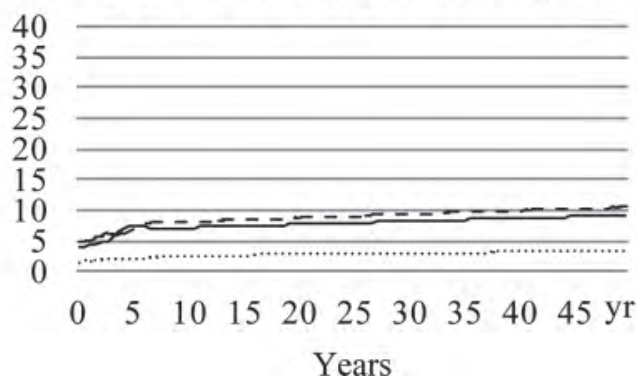

\section{L-LOPC with $20 \%$ Initial Infection}

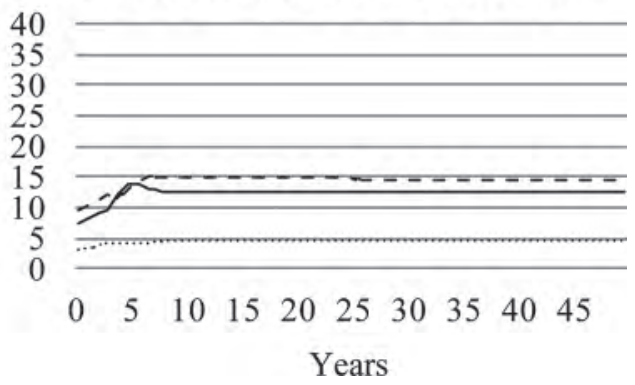

Figure 4. Effects of low-efficacy vaccines on the dynamics of Mycobacterium avium ssp. paratuberculosis (MAP) infection: portions of latent, low-shedding, and high-shedding cows in a herd of 100 cows and initial MAP prevalence of 10 and 20\% (vertical axis). L-S and H-S represent a low- (L-S) and high-efficacy (H-S) vaccine, in which the vaccine is targeted at decreasing susceptibility of susceptible calves; L-LC and H-LC represent a low- (L-LC) and high-efficacy (H-LC) vaccine, in which the vaccine is targeted at reducing shedding level and the number of clinical Johne's disease cases; L-OP and H-OP represent a low- (L-OP) and high-efficacy (H-OP) vaccine, in which the vaccine is targeted at delaying the onset of low shedding and slowing the progression from low to high shedding; L-LOPC and H-LOPC represent a low- (L-LOPC) and highefficacy (H-LOPC) vaccine, in which the vaccine is targeted at reducing MAP shedding level, delaying the onset of low shedding and progression from low to high shedding, and decreasing the number of clinical Johne's disease cases.

In this study, the NPV of a MAP-free farm $(0 \%$ initial infection level) was estimated to be $\$ 374,270$ for the 50 yr simulation period with a fixed herd size of 100 cows. On the other hand, the NPV of a MAP-infected farm was estimated to be considerably lower at $\$ 155,710$ (10\% initial infection level) and $\$ 88,819$ (20\% initial infection level) in the absence of MAP control. These low NPV values are not sustainable and imply that a farm 


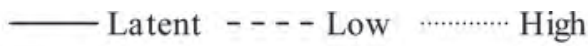

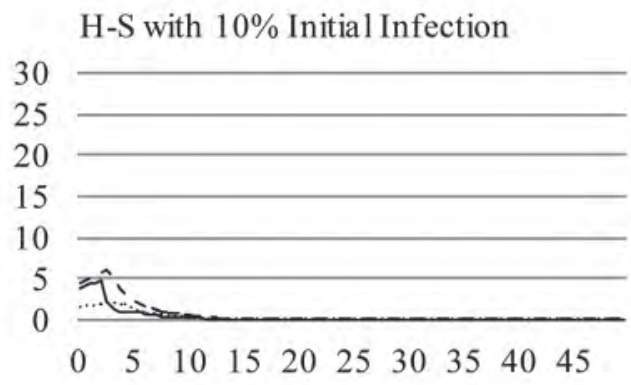
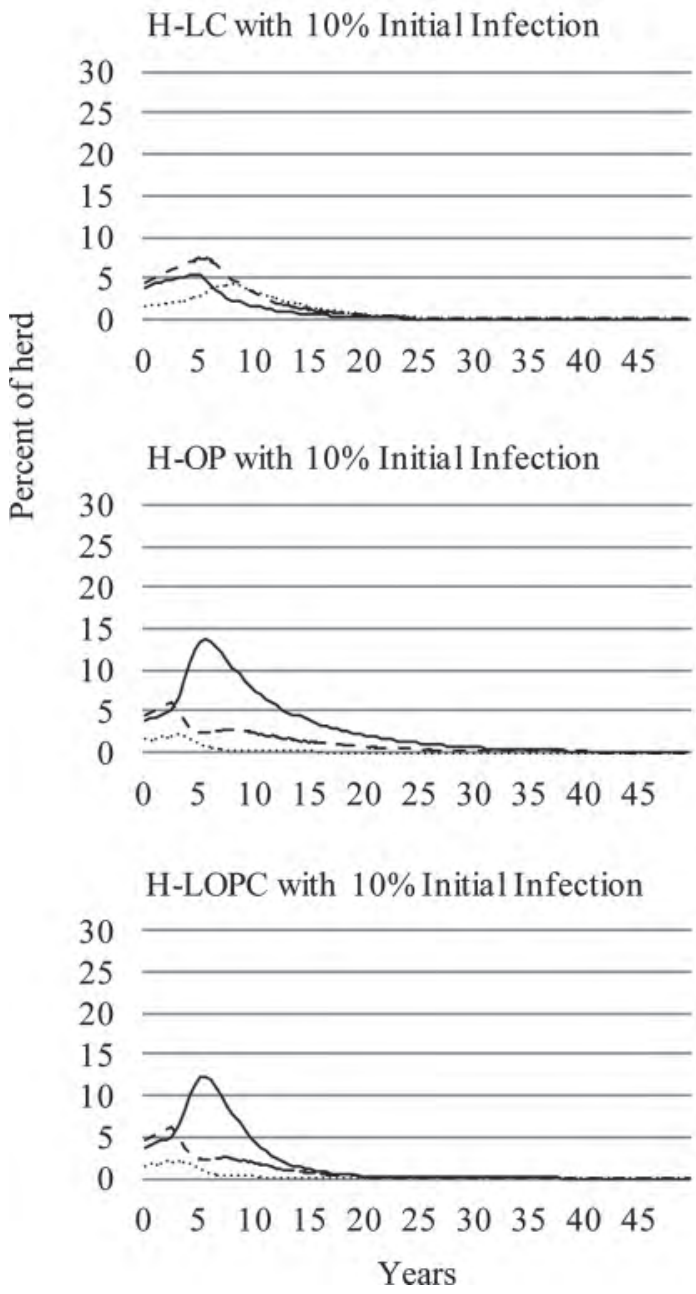

\section{H-S with $20 \%$ Initial Infection}

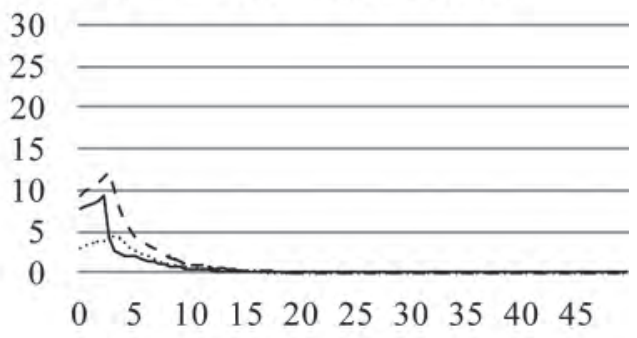

H-LC with $20 \%$ Initial Infection

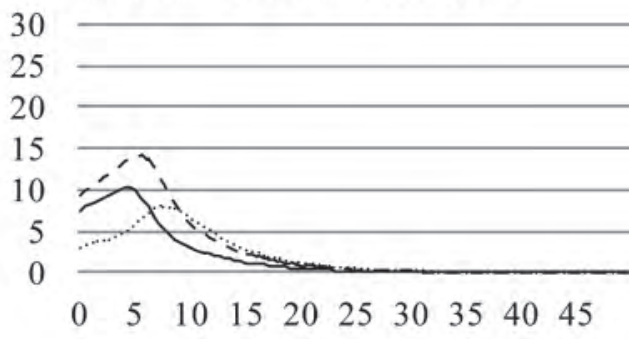

H-OP with $20 \%$ Initial Infection

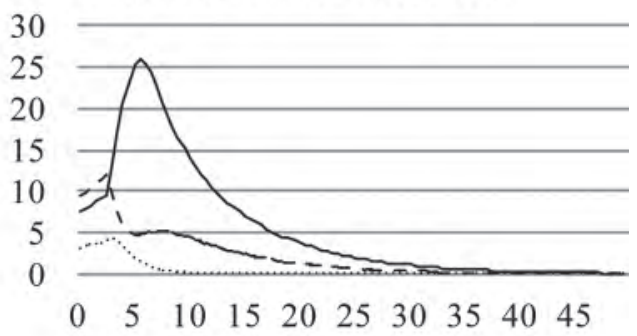

\section{H-LOPC with $20 \%$ Initial Infection}

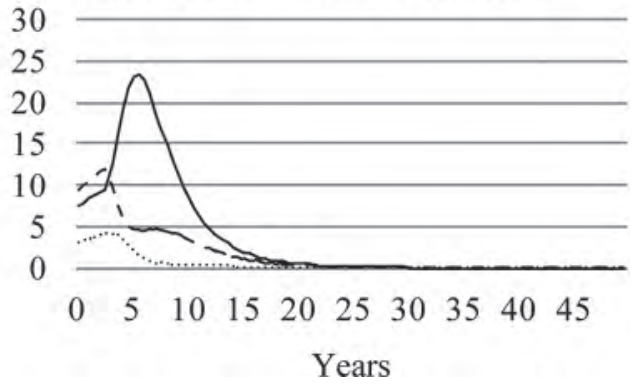

Figure 5. Effect of high-efficacy vaccines on the dynamics of Mycobacterium avium ssp. paratuberculosis (MAP) infection: portions of latent, low-shedding, high-shedding cows in a herd of 100 cows and initial MAP prevalence of 10 and 20\% (vertical axis). L-S and H-S represent a low- (L-S) and high-efficacy (H-S) vaccine, in which the vaccine is targeted at decreasing susceptibility of susceptible calves; L-LC and $\mathrm{H}-\mathrm{LC}$ represent a low- (L-LC) and high-efficacy (H-LC) vaccine, in which the vaccine is targeted at reducing shedding level and the number of clinical Johne's disease cases; L-OP and H-OP represent a low- (L-OP) and high-efficacy (H-OP) vaccine, in which the vaccine is targeted at delaying the onset of low shedding and slowing the progression from low to high shedding; L-LOPC and H-LOPC represent a low- (L-LOPC) and highefficacy (H-LOPC) vaccine, in which the vaccine is targeted at reducing MAP shedding level, delaying the onset of low shedding and progression from low to high shedding, and decreasing the number of clinical Johne's disease cases.

would eventually need to engage in some type of remedial action as the number of subclinical (low shedding) and clinical (high shedding) cows increased over time and before JD became pervasive in the herd. According to Cho et al. (2011), the best control alternative generated NPV of $\$ 345,603$ and $\$ 336,873$, which were 
MVC compared with no control with $10 \%$ initial infection

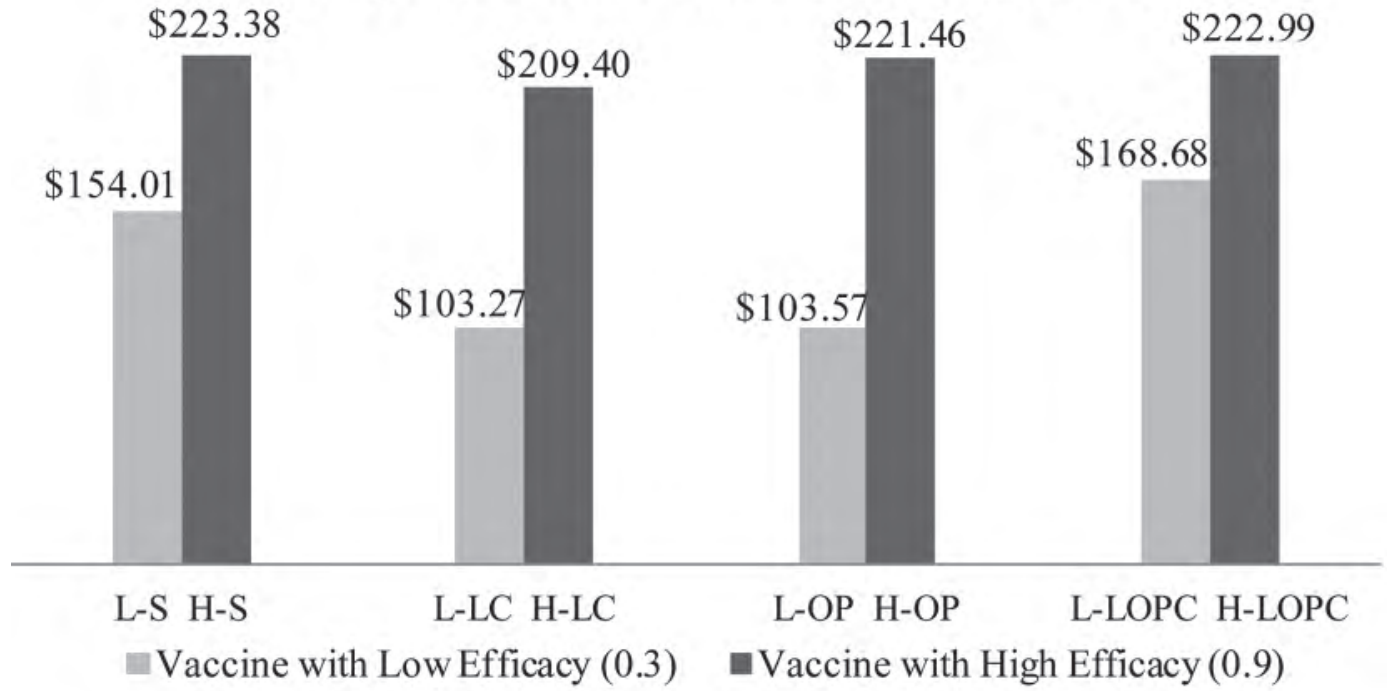

MVC compared with no control with $20 \%$ initial infection

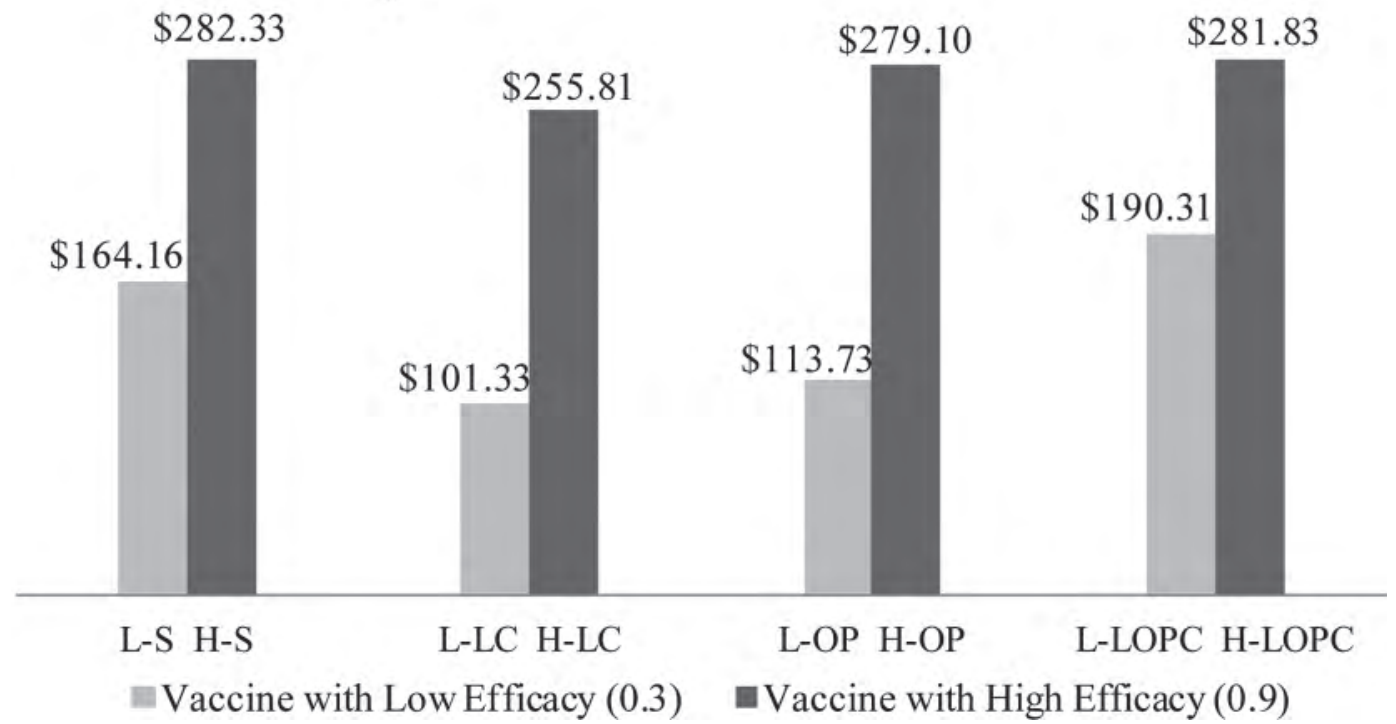

Figure 6. Maximum vaccination cost per dose (MVC) for Mycobacterium avium ssp. paratuberculosis (MAP) vaccine, which makes that vaccination option economically more attractive than no control when the initial MAP infection level is 10 or $20 \%$. L-S and $\mathrm{H}-\mathrm{S}$ represent a low- (L-S) and high-efficacy (H-S) vaccine, in which the vaccine is targeted at decreasing susceptibility of susceptible calves; L-LC and H-LC represent a low- (L-LC) and high-efficacy (H-LC) vaccine, in which the vaccine is targeted at reducing shedding level and the number of clinical Johne's disease cases; L-OP and H-OP represent a low- (L-OP) and high-efficacy (H-OP) vaccine, in which the vaccine is targeted at delaying the onset of low shedding and slowing the progression from low to high shedding; L-LOPC and H-LOPC represent a low- (L-LOPC) and highefficacy (H-LOPC) vaccine, in which the vaccine is targeted at reducing MAP shedding level, delaying the onset of low shedding and progression from low to high shedding, and decreasing the number of clinical Johne's disease cases.

significantly higher compared with the above NPV of a farm without control, given the initial infection levels of 10 and $20 \%$, respectively. These NPV were obtained when the herd size of 80 to 100 cows was assumed to allow heavy culling of test-positive animals under a test-and-cull strategy. With MAP vaccinations, the uppermost MVC for the $8 \mathrm{MAP}$ vaccination scenarios were standardized as the average present value per dose and are presented in Figures 6 and 7. These results suggest that any MAP vaccination would yield a higher NPV than that using the no-control alternative (Figure 6 ), and most of the high-efficacy vaccines had potential to generate a higher NPV than using the best alternative (Figure 7). 
Each MVC in Figures 6 and 7 was the maximum amount dairy producers would be willing to pay for implementing each vaccination instead of the nocontrol alternative or the best alternative control not involving vaccination. The high MVC in Figure 6 implies that any vaccination option has high potential to be greatly beneficial to dairy producers, compared with no control. In particular, 2 high-efficacy vaccines should be particularly attractive to dairy producers: (1) a vaccine that reduced susceptibility to MAP infection (scenario H-S), and (2) a vaccine that had multiple efficacies (scenario H-LOPC). This vaccine was targeted at reducing MAP shedding level, delaying the onset of shedding and progression from low to high shedding, and decreasing the number of clinical JD cases. These 2 scenarios in Figure 6 have MVC of $\$ 223.38$ (initial prevalence of $10 \%$ ) and $\$ 282.33$ (initial prevalence of $20 \%$ ), and $\$ 222.99$ (initial prevalence of $10 \%$ ) and $\$ 281.83$ (initial prevalence of $20 \%$ ), respectively. These MVC imply that JD inflicts an annual cost of $\$ 96.03$ $(\$ 222.99 \times 43 \div 100)$ to $\$ 121.18(=\$ 282.33 \times 43 \div$ 100) per cow in the herd, where 43 is the number of vaccinated calves per year and 100 is the number of cows in the herd. These 2 high-efficacy vaccines also have the highest MVC of $\$ 17.79$ (initial prevalence of $10 \%$ ) and $\$ 13.77$ (initial prevalence of $20 \%$ ), and $\$ 17.40$ (initial prevalence of $10 \%$ ) and $\$ 13.27$ (initial prevalence of $20 \%$ ) compared with the best alternative, respectively (Figure 7). Considering that these 2 highefficacy vaccinations were the most effective (scenario $\mathrm{H}-\mathrm{S}$ ) and the second most effective (scenario H-LOPC) vaccination options in controlling MAP, as discussed in the previous section, a high-efficacy vaccine that reduces susceptibility (scenario H-S) might be the best vaccination option overall, followed by a high-efficacy vaccine with multiple efficacies on the dynamics of MAP infection and progress (scenario H-LOPC).

In the bottom of Figure 7, only one high-efficacy vaccine described in scenario H-LC had a negative MPV of $-\$ 12.75$, which implies that this vaccination always generates a lower NPV than that using the best alternative, given an initial infection level of $20 \%$. This implies that the vaccine in scenario H-LC, in which the vaccine is targeted at reducing MAP shedding level and the number of clinical JD cases, may not be economically beneficial to dairy producers when their herds are highly infected with MAP. This result also implies that many of the currently available MAP vaccines may not be economically attractive compared with the best alternative of improved hygiene management and testand-cull using an annual fecal culture test, when the herd is highly infected with MAP. This is because the vaccine in scenario $\mathrm{H}-\mathrm{LC}$ was designed to represent the commonly reported effects of currently available MAP vaccines, which reduces MAP shedding level and the number of clinical JD cases. In addition, the negative MVC associated with the low-efficacy vaccine scenarios (Figure 7) suggest that none of the low-efficacy vaccine scenarios can generate a higher NPV compared with the best alternative, given the same infection levels, and therefore, they may not be an economically more attractive control method than the best alternative. Nevertheless, low-efficacy vaccines have high potential to generate a higher NPV compared with the no-control strategy, given that their MVC values are significantly positive for all scenarios, as shown in Figure 6.

The MVC values discussed above, however, are maximum values based upon breakeven estimates, and do not reflect profit sharing with vaccine developers and do not take risk considerations into account. Therefore, even though a vaccination has a positive MVC, it may or may not be an economically better MAP control method compared with either alternative, because market prices for these hypothetical vaccines are not known at this time. For example, the maximum MVC for the high-efficacy vaccine in scenario $\mathrm{H}-\mathrm{S}$ is reported in Figure 7 as $\$ 17.79$, given the initial infection level of $10 \%$. However, this MVC is not the potential economic benefit of the vaccination for a dairy producer relative to the best alternative. In fact, this number represents the potential maximum gross return from the vaccination, which would be shared by a dairy producer (profit and vaccination cost if any) and a vaccine company (profit and production cost). Therefore, whether or not this vaccination would generate a higher economic benefit, in terms of NPV, for a dairy producer, than the best alternative is inconclusive given the lack of information on the actual cost of the vaccine to the dairy producer. Nevertheless, this vaccination could generate a higher NPV of $\$ 362,034$ and $\$ 349,592$ compared with using the best alternative $(\$ 345,603$ and $\$ 336,873)$ given initial infection levels of 10 and $20 \%$, respectively, when the cost of the vaccination to the dairy producer is zero.

Although the MVC of the various vaccination scenarios are presented in Figures 6 and 7, it is useful to convert these into maximum feasible NPV of vaccination (Table 6). Maximum feasible NPV is the NPV with the vaccination compared with either no control or the best alternative when the cost of the vaccination is zero. Given a constant herd size of 100 cows and a birth rate of female calves of 0.215 per $6 \mathrm{mo}$, the maximum feasible annual economic benefit associated with vaccination compared with either no control or the best alternative can be calculated by multiplying a MVC value for a MAP vaccine times $43(100 \times 0.215$ $\times 2$ ), which is the number of vaccinated calves per year. Maximum feasible annual economic benefit is the annual economic benefit with the vaccination compared 


\section{MVC compared with the best alternative with $10 \%$ initial infection}

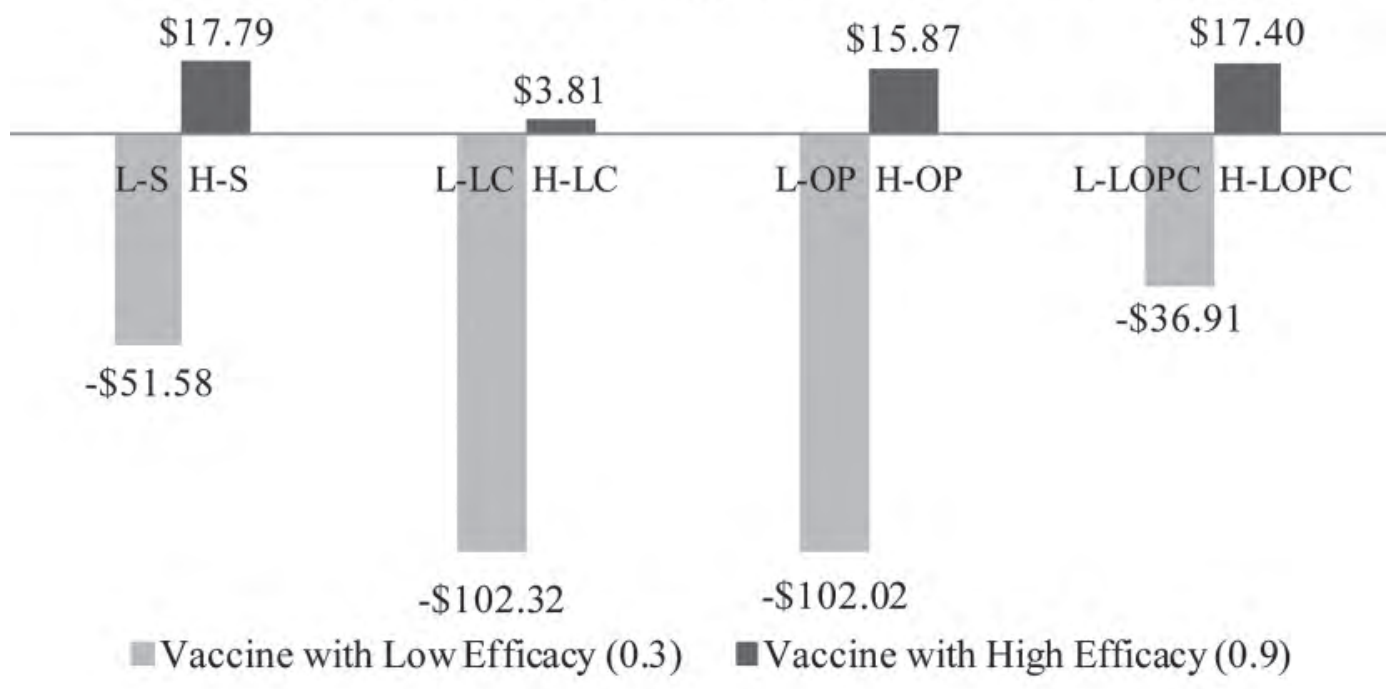

MVC compared with the best alternative with $20 \%$ initial infection

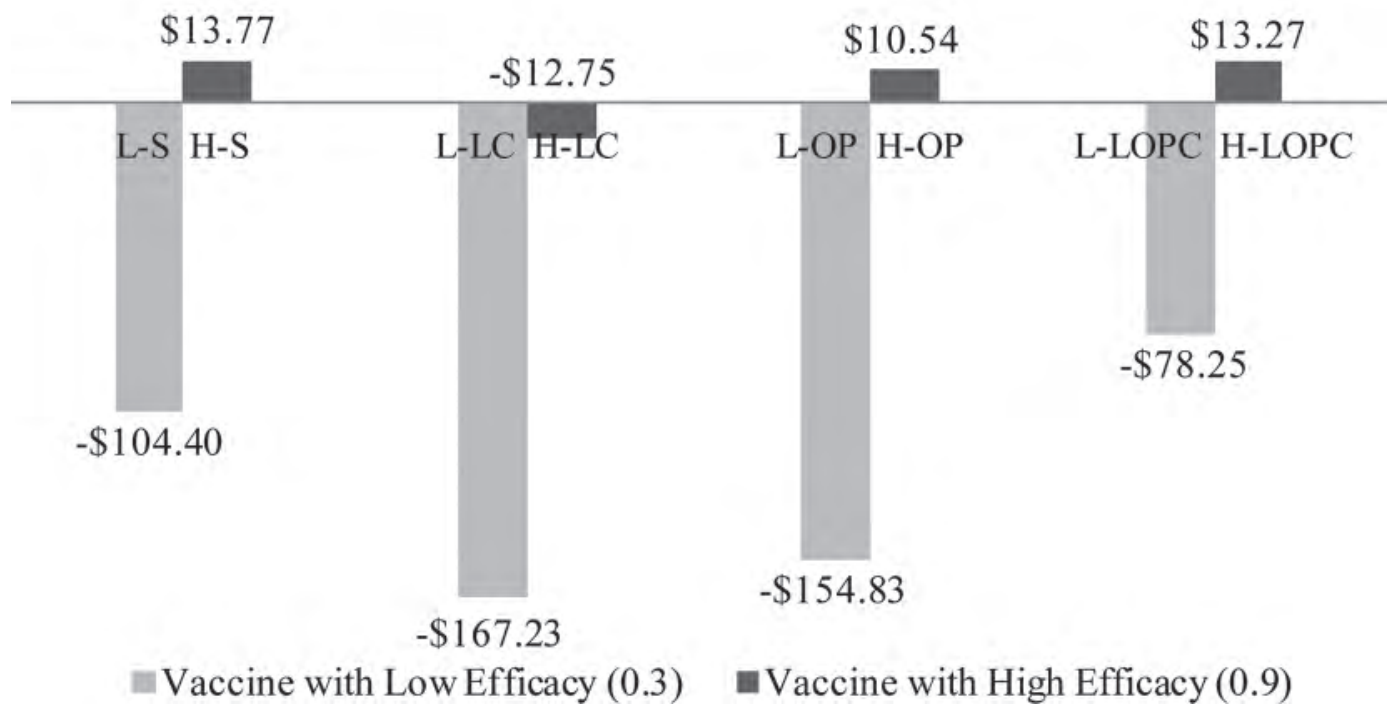

Figure 7. Maximum vaccination cost per dose (MVC) for Mycobacterium avium ssp. paratuberculosis (MAP) vaccine, which makes that vaccination option economically more attractive than the best alternative (improved hygiene management and test-and-cull using an annual fecal culture test) when the initial MAP infection level is 10 or $20 \%$. L-S and H-S represent a low- (L-S) and high-efficacy (H-S) vaccine, in which the vaccine is targeted at decreasing susceptibility of susceptible calves; L-LC and H-LC represent a low- (L-LC) and high-efficacy (H-LC) vaccine, in which the vaccine is targeted at reducing shedding level and the number of clinical Johne's disease cases; L-OP and H-OP represent a low- (L-OP) and high-efficacy (H-OP) vaccine, in which the vaccine is targeted at delaying the onset of low shedding and slowing the progression from low to high shedding; L-LOPC and H-LOPC represent a low- (L-LOPC) and high-efficacy (H-LOPC) vaccine, in which the vaccine is targeted at reducing MAP shedding level, delaying the onset of low shedding and progression from low to high shedding, and decreasing the number of clinical Johne's disease cases.

with either no control or the best alternative when the cost of the vaccination is zero. Positive value represents benefit, whereas negative value represents loss. Adding the NPV of either no control or the best alternative to this annual economic benefit associated with vaccination represents the maximum feasible NPV of vaccination. The high-efficacy vaccination that decreased susceptibility of susceptible calves has the highest MVC value (scenario H-S), and could generate NPV of $\$ 362,034$ and $\$ 349,592$, which are significantly higher compared with a farm without control with NPV of $\$ 155,710$ and $\$ 88,819$, given the initial infection level of $10 \%$ and $20 \%$, respectively. These NPV are also higher compared with those on a farm employing the best 
Table 6. Maximum feasible net present value (NPV) of vaccination given an initial Mycobacterium avium ssp. paratuberculosis (MAP) infection level of $10 \%$ and $20 \%$

\begin{tabular}{lcc}
\hline & \multicolumn{2}{c}{ Maximum feasible $\mathrm{NPV}^{2}$ of vaccination } \\
\cline { 2 - 3 } Scenario $^{1}$ & $\begin{array}{c}\text { Infection level } \\
\text { of } 10 \%\end{array}$ & $\begin{array}{c}\text { Infection level } \\
\text { of } 20 \%\end{array}$ \\
\hline L-S & $\$ 297,961$ & $\$ 240,445$ \\
H-S & $\$ 362,034$ & $\$ 349,592$ \\
L-LC & $\$ 251,095$ & $\$ 182,412$ \\
H-LC & $\$ 349,122$ & $\$ 325,097$ \\
L-OP & $\$ 251,372$ & $\$ 193,865$ \\
H-OP & $\$ 360,261$ & $\$ 346,609$ \\
L-LOPC & $\$ 311,511$ & $\$ 264,598$ \\
H-LOPC & $\$ 361,674$ & $\$ 349,130$ \\
\hline
\end{tabular}

${ }^{1}$ The notation L-S and H-S represent a low- (L-S) and high-efficacy (H-S) vaccine, in which the vaccine is targeted at decreasing susceptibility of susceptible calves. L-LC and H-LC represent a low- (L-LC) and high-efficacy (H-LC) vaccine, in which the vaccine is targeted at reducing shedding level and the number of clinical Johne's disease cases. L-OP and H-OP represent a low- (L-OP) and high-efficacy $(\mathrm{H}-$ $\mathrm{OP}$ ) vaccine, in which the vaccine is targeted at delaying the onset of low shedding and slowing the progression from low to high shedding. L-LOPC and H-LOPC represent a low- (L-LOPC) and high-efficacy (H-LOPC) vaccine, in which the vaccine is targeted at reducing MAP shedding level, delaying the onset of low shedding and progression from low to high shedding, and decreasing the number of clinical Johne's disease cases. ${ }^{2}$ Maximum feasible NPV is the NPV with the vaccination compared with either no control or the best alternative when the cost of the vaccination is zero.

alternative (NPV of $\$ 345,603$ and $\$ 336,873$ ). These NPV were obtained when heavy culling of test-positive animals was allowed in the herds of 80 to 100 cows from our previous study for maximizing NPV.

\section{CONCLUSIONS}

This study evaluated both the epidemiological consequences and economic value of various MAP vaccines in dairy herds, using a discrete dynamic model that incorporated dynamics of MAP transmission within a herd and maximized NPV of the farm's cash flow over a planning duration. Eight vaccination scenarios, which comprised various combinations of vaccine efficacies, were developed to represent the potential impact on the epidemiological process of MAP of different types of vaccines. These scenarios allowed us to compare different epidemiological effects of current and nextgeneration vaccines on MAP control. Elimination of MAP requires a long-term plan with implementation of at least one of the high-efficacy vaccines. Any vaccination with a low- or high-efficacy vaccine yielded a higher NPV compared with no control when the cost of these vaccinations was less than their MVC. Any highefficacy vaccine, with the exception of the vaccine targeted at reducing MAP shedding level and the number of clinical JD cases (scenario H-LC), generated an even higher NPV compared with a farm with the best alter- native when the cost of these vaccinations was less than their MVC. Given the epidemiological consequences and economic benefits of various vaccination options, a high-efficacy vaccine that reduces susceptibility (scenario $\mathrm{H}-\mathrm{S}$ ) might be the best vaccination option, and a high-efficacy vaccine that has multiple efficacies on the dynamics of MAP infection and progress (scenario H-LOPC) might be the second best vaccination option for dairy producers in MAP control.

\section{ACKNOWLEDGMENTS}

The authors gratefully acknowledge funding provided by the USDA-CSREES award number 2008-3520404627 and the Johne's Disease Integrated Program (JDIP, USDA) contract number 45105.

\section{REFERENCES}

Aly, S. S., R. J. Anderson, J. M. Adaska, J. Jiang, and I. A. Gardner. 2010. Association between Mycobacterium avium subspecies paratuberculosis infection and milk production in two California dairies. J. Dairy Sci. 93:1030-1040.

Benedictus, A., R. Mitchell, M. Linde-Widmann, R. Sweeney, T. Fyock, Y. Schukken, and R. Whitlock. 2008. Transmission parameters of Mycobacterium avium subspecies paratuberculosis infections in a dairy herd going through a control program. Prev. Vet. Med. 83:215-227.

Cho, J., L. Tauer, Y. Schukken, R. Smith, Z. Lu, and Y. Gröhn. 2011. Compartment model for controlling infectious livestock disease: Cost-effective control strategies for Johne's disease in dairy herds. Paper in Proc. 2011 Agric. Appl. Econ. Assoc. Annu. Mtg. Agric. Appl. Econ. Assoc., Pittsburgh, PA. Accessed Jun. 30, 2011. http://purl.umn.edu/103393.

Collins, M. T., V. Eggleston, and E. Manning. 2010. Successful control of Johne's disease in nine dairy herds: Results of a six-year field trial. J. Dairy Sci. 93:1638-1643.

Collins, M. T., and I. Morgan. 1991. Economic decision analysis model of a paratuberculosis test and cull program. J. Am. Vet. Med. Assoc. 199:1724-1729.

Collins, M. T., I. A. Gardner, F. B. Garry, A. J. Roussel, and S. J. Wells. 2006. Consensus recommendations on diagnostic testing for the detection of paratuberculosis in cattle in the United States. J. Am. Vet. Med. Assoc. 229:1912-1919.

Dorshorst, N. C., M. Collins, and J. Lombard. 2006. Decision analysis model for paratuberculosis control in commercial dairy herds. Prev. Vet. Med. 75:92-122.

Feller, M., K. Huwiler, R. Stephan, E. Altpeter, A. Shang, H. Furrer, G. E. Pfyffer, T. Jemmi, A. Baumgartner, and M. Egger. 2007. Mycobacterium avium subspecies paratuberculosis and Crohn's disease: A systematic review and meta-analysis. Lancet Infect. Dis. 7:607-613.

Groenendaal, H., and D. Galligan. 2003. Economic consequences of control programs for paratuberculosis in midsize dairy farms in the United States. J. Am. Vet. Med. Assoc. 223:1757-1763.

Groenendaal, H., M. Nielen, A. Jalvingh, S. Horst, D. Galligan, and J. Hesselink. 2002. A simulation of Johne's disease control. Prev. Vet. Med. 54:225-245.

Groenendaal, H., and F. J. Zagmutt. 2008. Scenario analysis of changes in consumption of dairy products caused by a hypothetical causal link between Mycobacterium avium subspecies paratuberculosis and Crohn's disease. J. Dairy Sci. 91:3245-3258.

Harris, N. B., and R. G. Barletta. 2001. Mycobacterium avium ssp. paratuberculosis in veterinary medicine. Clin. Microbiol. Rev. $14: 489-512$. 
Hermon-Taylor, J. 2009. Mycobacterium avium subspecies paratuberculosis, Crohn's disease and the Doomsday scenario. Gut Pathog. $1: 15$.

Kalis, C. H., J. W. Hesselink, H. W. Barkema, and M. T. Collins. 2001. Use of long-term vaccination with a killed vaccine to prevent fecal shedding of Mycobacterium avium subsp paratuberculosis in dairy herds. Am. J. Vet. Res. 62:270-274.

Karszes, J., C. Wickswat, and F. Vokey. 2008. Dairy Replacement Programs: Costs \& Analysis. December 2007. Cornell University. Accessed May 30, 2010. http://dyson.cornell.edu/outreach/extensionpdf/2008/Cornell_AEM_eb0816.pdf.

Keeble, J. R., and K. Walker. 2009. Therapeutic vaccine comprising Mycobacterium HSP70. Expert Opin Ther. Pat. 19:95-99.

Koets, A., A. Hoek, M. Langelaar, M. Overdijk, W. Santema, P. Franken, W. Eden, and V. Rutten. 2006. Mycobacterial $70 \mathrm{kD}$ heatshock protein is an effective subunit vaccine against bovine paratuberculosis. Vaccine 24:2550-2559.

Köhler, H., H. Gyra, K. Zimmer, K. Dräger, B. Burkert, B. Lemser, D. Hausleithner, K. Cussler, W. Klawonn, and R. Hess. 2001. Immune reactions in cattle after immunization with a Mycobacterium paratuberculosis vaccine and implications for the diagnosis of $M$. paratuberculosis and M. bovis infections. J. Vet. Med. B 48:185-195.

Kudahl, A. B., and S. S. Nielsen. 2009. Effect of paratuberculosis on slaughter weight and slaughter value of dairy cows. J. Dairy Sci. 92:4340-4346.

Lu, Z., R. Mitchell, R. Smith, J. Van Kessel, P. Chapagain, Y. Schukken, and Y. Grohn. 2008. The importance of culling in Johne's disease control. J. Theor. Biol. 254:135-146.

Lu, Z., Y. Schukken, R. Smith, and Y. Grohn. 2010. Stochastic simulations of a multi- group compartmental model for Johne's disease on US dairy herds with test-based culling intervention. J. Theor. Biol. 264:1190-1201.

Mitchell, R. M., R. Whitlock, S. Stehman, A. Benedictus, P. Chapagain, Y. Grohn, and Y. Schukken. 2008. Simulation modeling to evaluate the persistence of Mycobacterium avium ssp. paratuberculosis (MAP) on commercial dairy farms in the United States. Prev. Vet. Med. 83:360-380.

Muskens, J., F. Van Zijderveld, A. Eger, and D. Bakker. 2002. Evaluation of the long-term immune response in cattle after vaccination against paratuberculosis in two Dutch dairy herds. Vet. Microbiol. 86:269-278.

Ott, S. L., S. Wells, and B. Wagner. 1999. Herd-level economic losses associated with Johne's disease on US dairy operations. Prev. Vet. Med. 40:179-192.

Pillars, R. B., D. Grooms, C. Wolf, and J. Kaneene. 2009. Economic evaluation of Johne's disease control programs implemented on six Michigan dairy farms. Prev. Vet. Med. 90:223-232.

Raizman, E. A., J. P. Fetrow, and S. J. Wells. 2009. Loss of income from cows shedding Mycobacterium avium subspecies paratuberculosis prior to calving compared with cows not shedding the organism on two Minnesota dairy farms. J. Dairy Sci. 92:4929-4936.

Rankin, J. D. 1961. The experimental infection of cattle with Mycobacterium johnei. III. Calves maintained in an infectious environment. J. Comp. Pathol. 71:10-15.

Romano, M., and K. Huygen. 2009. DNA vaccines against mycobacterial diseases. Expert Rev. Vaccines 8:1237-1250.

Rosseels, V., and K. Huygen. 2008. Vaccination against paratuberculosis. Expert Rev. Vaccines 7:817-832.

Rosseels, V., S. Marche, V. Roupie, M. Govaerts, J. Godfroid, K. Walravens, and K. Huygen. 2006. Members of the 30- to 32-kilodalton mycolyl transferase family (Ag85) from culture filtrate of Mycobacterium avium ssp. paratuberculosis are immunodominant Th1-type antigens recognized early upon infection in mice and cattle. Infect. Immun. 74:202-212.

Smith, R. L., Y. Grohn, A. Pradhan, R. Whitlock, J. Van Kessel, J. Smith, D. Wolfgang, and Y. Schukken. 2009. A longitudinal study on the impact of Johne's disease status on milk production in individual cows. J. Dairy Sci. 92:2653.
Sorge, U., D. Kelton, K. Lissemore, A. Godkin, S. Hendrick, and S. Wells. 2010. Attitudes of Canadian dairy farmers toward a voluntary Johne's disease control program. J. Dairy Sci. 93:1491-1499.

Sweeney, R. W., R. Whitlock, and A. Rosenberger. 1992a. Mycobacterium paratuberculosis cultured from milk and supramammary lymph nodes of infected asymptomatic cows. J. Clin. Microbiol. 30:166.

Sweeney, R. W., R. Whitlock, and A. Rosenberger. 1992b. Mycobacterium paratuberculosis isolated from fetuses of infected cows not manifesting signs of the disease. Am. J. Vet. Res. 53:477.

Sweeney, R. W. 1996. Transmission of paratuberculosis. Vet. Clin. North Am. Food Anim. Pract. 12:305.

USDA. 1997. Johne's disease on U.S. Dairy Operations. Animal and Plant Health Inspection Service, Washington DC. Accessed May 30, 2010. http://purl.umn.edu/32763.

USDA. 2007. Dairy 2007. Part I: Reference of Dairy Cattle Health and Management Practices in the United States, 2007. Animal and Plant Health Inspection Service, Washington, DC. Accessed May 30, 2010. http://www.aphis.usda.gov/animal_health/nahms/ dairy/downloads/dairy07/Dairy0 7_dr_PartI.pdf.

USDA. 2008. Johne's Disease on U.S. Dairies, 1991-2007. Animal and Plant Health Inspection Service, Washington, DC. Accessed May 30, 2010. http://www.aphis.usda.gov/animal_health/nahms/ dairy/downloads/dairy07/Dairy0 7_is_Johnes.pdf.

USDA. 2010. Questions and Answers: Bovine Tuberculosis Federal Order. Animal and Plant Health Inspection Service, Washington, DC. Accessed Jan. 15, 2011. http://www.aphis.usda.gov/publications/animal_health/content/printable_version/f aq_bovine_tb_ fed_order.pdf.

USDA. 2003-2007. Agricultural Prices Summary 2003-2007. Accessed May 30, 2010. http://usda.mannlib.cornell.edu/MannUsda/viewDocumentInfo.do?documentID=1003.

van Schaik, G., C. Kalis, G. Benedictus, A. Dijkhuizen, and R. Huirne. 1996. Cost-benefit analysis of vaccination against paratuberculosis in dairy cattle. Vet. Rec. 139:624.

van Schaik, G.. C. R. Rossiter, S. M. Stehman, S. J. Shin, and Y. H. Schukken. 2003a. Longitudinal study to investigate variation in results of repeated ELISA and culture of fecal samples for Mycobacterium avium subsp. paratuberculosis in commercial dairy herds. Am. J. Vet. Res. 64:479-484.

van Schaik, G., Y. Schukken, C. Crainiceanu, J. Muskens, and J. VanLeeuwen. 2003b. Prevalence estimates for paratuberculosis adjusted for test variability using Bayesian analysis. Prev. Vet. Med. 60:281-295.

Waddell, L. A., A. Raji, J. Sargeant, J. Harris, R. Amezcua, L. Downey, S. Read, and S. A. McEwen. 2008. The zoonotic potential of Mycobacterium avium ssp. paratuberculosis: A systematic review. Can. J. Public Health 99:145-155.

Wells, S. J., R. Whitlock, B. Wagner, J. Collins, F. Garry, H. Hirst, J. Lawrence, W. Saville, and A. Naugle. 2002. Sensitivity of test strategies used in the Voluntary Johne's Disease Herd Status Program for detection of Mycobacterium paratuberculosis infection in dairy cattle herds. J. Am. Vet. Med. Assoc. 220:1053-1057.

Whitlock, R., R. Sweeney, T. Fyock, and J. Smith. 2005a. MAP supershedders: Another factor in the control of Johne's disease. Page 164 in Proc. 8th Int. Colloq. Paratuberculosis, Copenhagen, Denmark.

Whitlock, R. H., S. Wells, R. Sweeney, and J. Van Tiem. 2000. ELISA and fecal culture for paratuberculosis (Johne's disease): Sensitivity and specificity of each method. Vet. Microbiol. 77:387-398.

Whitlock, R., M. Widmann, R. Sweeney, T. Fyock, M. Linde, A. Benedictus, R. Mitchell, and Y. Schukken. 2005b. Estimation of parameters on the vertical transmission of Map in a low-prevalence dairy herd. Page 706 in Proc. 8th Int. Colloq. Paratuberculosis, Copenhagen, Denmark.

Whitlock, R. H., and C. Buergelt. 1996. Preclinical and clinical manifestations of paratuberculosis (including pathology). Vet. Clin. North Am. Food Anim. Pract. 12:345. 


\section{APPENDIX}

The dynamics of MAP transmission within a herd described in the animal compartment model of Figure 1 are represented by the following equations of motion:

$$
\begin{gathered}
X_{1}(t+1)=(1-p)\left[\mu_{b}-\gamma(t)\right]\left\{\sum_{i=11}^{14}\left[X_{i}(t)+V_{i}(t)\right]\right\} \\
+\left\{1-\left[\lambda(t)+\mu_{c}+\chi(t)\right]\right\} X_{1}(t), \\
X_{2}(t+1)=(1-p) \gamma(t)+\left[1-\left(\mu_{c}+\rho_{c}\right)\right] X_{2}(t), \\
X_{3}(t+1)=\chi(t) X_{1}(t)+\left\{1-\left[\lambda(t)+\mu_{c}+\chi(t)\right]\right\} X_{3}(t), \\
X_{4}(t+1)=\lambda(t) X_{1}(t)+\left[1-\left(\mu_{c}+\rho_{c}\right)\right] X_{4}(t), \\
X_{5}(t+1)=\rho_{c} X_{2}(t)+\left[1-\left(\mu_{c}+\rho_{c}\right)\right] X_{5}(t), \\
X_{6}(t+1)=\chi(t) X_{3}(t)+\left[1-\left(\mu_{h}+\rho_{h}\right)\right] X_{6}(t), \\
X_{7}(t+1)=\lambda(t) X_{3}(t)+\left[1-\left(\mu_{h}+\rho_{h}\right)\right] X_{7}(t), \\
X_{8}(t+1)=\rho_{c}\left[X_{4}(t)+X_{5}(t)\right]+\left[1-\left(\mu_{h}+\rho_{h}\right)\right] X_{8}(t), \\
X_{1}(t+1)=p\left[\mu_{b}-\gamma(t)\right]\left\{\sum_{i=11}\left[X_{i}(t)+V_{i}(t)\right]\right\} \\
X_{14}(t+1)=v X_{13}(t)+\left\{1-\left[\mu_{a}(t)+\alpha\right]\right\} X_{14}(t), \\
X_{11}(t+1)=\rho_{h} X_{6}(t)+\left[1-\left(\mu_{h}+\rho_{h}\right)\right] X_{9}(t), \\
\left.\left.X_{10}(t+1)\right]\right\} V_{1}(t),
\end{gathered}
$$

$$
\begin{gathered}
V_{2}(t+1)=p \gamma(t)+\left[1-\left(\mu_{c}+\rho_{c}\right)\right] V_{2}(t), \\
V_{3}(t+1)=\varepsilon(t) V_{1}(t) \\
+\left\{1-\left[\left(1-e_{\lambda}\right) \lambda(t)+\mu_{c}+\varepsilon(t)\right]\right\} V_{3}(t), \\
V_{4}(t+1)=\left(1-e_{\lambda}\right) \lambda(t) V_{1}(t)+\left[1-\left(\mu_{c}+\rho_{c}\right)\right] V_{4}(t), \\
V_{5}(t+1)=\rho_{c} V_{2}(t)+\left[1-\left(\mu_{c}+\rho_{c}\right)\right] V_{5}(t), \\
V_{6}(t+1)=\varepsilon(t) V_{3}(t)+\left[1-\left(\mu_{h}+\rho_{h}\right)\right] V_{6}(t), \\
V_{7}(t+1)=\left(1-e_{\lambda}\right) \lambda(t) V_{3}(t)+\left[1-\left(\mu_{h}+\rho_{h}\right)\right] V_{7}(t), \\
V_{8}(t+1)=\rho_{c}\left[V_{4}(t)+V_{5}(t)\right]+\left[1-\left(\mu_{h}+\rho_{h}\right)\right] V_{8}(t) \\
\quad+\left\{1-\left[\mu_{a}(t)+\left(1-e_{v}\right) v\right]\right\} V_{13}(t), \\
V_{9}(t+1)=\rho_{h} V_{6}(t)+\left[1-\left(\mu_{h}+\rho_{h}\right)\right] V_{9}(t), \\
V_{10}(t+1)=\rho_{h}\left[V_{7}(t)+V_{8}(t)\right]+\left[1-\left(\mu_{h}+\rho_{h}\right)\right] V_{10}(t), \\
V_{13}(t+1)=\left(1-e_{v}\right) v V_{13}(t) \\
V_{11}(t+1)=\rho_{h} V_{9}(t)+\left[1-\mu_{a}(t)\right] V_{11}(t), \\
\end{gathered}
$$

where the definitions of parameters and transition rates are presented in Tables 1 and 2 and the vertical $(\gamma)$ and horizontal $(\lambda)$ MAP transmission rates are described in equations [1] and [2], respectively. 